\title{
Stereokinetic Effect and Its Relation to the Kinetic Depth Effect
}

\author{
Dennis R. Proffitt \\ University of Virginia \\ Heiko Hecht \\ University of Virginia
}

\author{
Irvin Rock
of California, \\ University of California, Berkeley \\ Jim Schubert \\ Rutgers University
}

\begin{abstract}
The stereokinetic effect (SKE) has been defined and studied by nested circular patterns rotating on a turntable. Circles must appear not to rotate as they revolve, which in turn results in their appearing to translate relative to one another. A powerful illusion of object depth results even though the individual circles do not undergo an appropriate foreshortening consistent with their apparent changes in slant. It is suggested and tested that the SKE is based on the changing positions between the nested contours despite the absence of any change within each contour, whereas the kinetic depth effect (KDE) entails both kinds of change. It follows that a turntable method of presentation is not necessary, and between-contour transformations can be simulated by computer animation. Displays consisting of simple translations were shown to evoke robust depth impressions as were patterns consisting of contours of varying shapes. Comparisons of the depth, compellingness, and rigidity of matched SKE and KDE displays are reported. The SKE is taken to be paradigmatic for how the visual system perceives depth when observing small object rotations that occur in everyday situations.
\end{abstract}

Whenever a rigid object is observed to rotate about some axis other than the observer's line of sight, optical transformations occur from which the object's three-dimensional configuration can be extracted. Typically, human sensitivities to this information have been investigated by having observers look at two-dimensional projections (polar or orthographic) of rotating forms, and in such situations the accurate perception of rigid objects rotating in depth is quite robust. Following Wallach and O'Connell (1953), we refer to this perception of three-dimensional form when viewing projections of rotating objects as the kinetic depth effect (KDE).

In this article, we show that the motions present in the $\mathrm{KDE}$ can be decomposed into two distinct transformations having quite different perceptual significances. The first of these transformations defines the stimulus conditions for the stereokinetic effect (SKE); when observed in isolation, it evokes the perception of a definite rigid form even though in a strict geometrical sense the manifest motion patterns do not fully support such a percept. The second transformation can also be isolated, and when these motions are observed using multiple nested contours, an elastic surface having little depth is seen. It is shown that the former transformation specifies depth relations within three-dimensional objects, whereas the latter relates more to the orientation of surfaces relative to the

This research was supported by Air Force Office of Scientific Research Grant AFOSR-91-0057 and National Aeronautics and Space Administration Grant NCA2-468 to Dennis R. Proffitt and by National Institute of Mental Health Research Scientist Award K05 MH00707 to Irvin Rock. All computer graphics were programmed by Steve Jacquot.

Correspondence concerning this article should be sent to Dennis R. Proffitt, Department of Psychology, Gilmer Hall, University of Virginia, Charlottesville, Virginia 22903-2477. observer. Before discussing these transformations in detail, some background on the SKE is provided.

\section{Stereokinetic Effect}

Musatti (1924) published the first report on stereokinetic phenomena and attributed their discovery and naming to his teacher, Vittorio Benussi. In his early writings, Musatti referred to all depth perceptions that were based on motion information as stereokinetic phenomena. Thus, three classes of events were considered by him to be examples of the SKE: (a) perceiving depth from motion parallax, (b) what we now refer to as the $\mathrm{KDE}$, and (c) depth impressions evoked by certain two-dimensional patterns that have been rotated in the picture plane. Later Musatti (1975) restricted the definition of the SKE to the latter class of events, and this use of the term has become conventional.

By far the most frequently studied SKE pattern was introduced by Musatti (1924) and is shown in the top panel of Figure 1. The pattern consists of a set of nested circles having a constant eccentricity. When this pattern is placed on a vertical turntable and slowly rotated, observers spontaneously perceive a three-dimensional form appearing as either a cone pointing outward or as a funnel receding inward. These are two depth percepts that often spontaneously reverse. For convenience, we refer to this pattern as the SKE cone.

It may seem odd that a two-dimensional pattern rotating in the picture plane should evoke the perception of a threedimensional solid; however, certain factors mediate against the two-dimensional percept. As is shown in the middle and bottom panels of Figure 1, the motions of the circular contours are ambiguous. Because the circular contours are smooth, motion in the direction of the contours may not be detectable, in which case only those motions that are orthogonal to the contours will be apparent (Hildreth, 1984; Wal- 

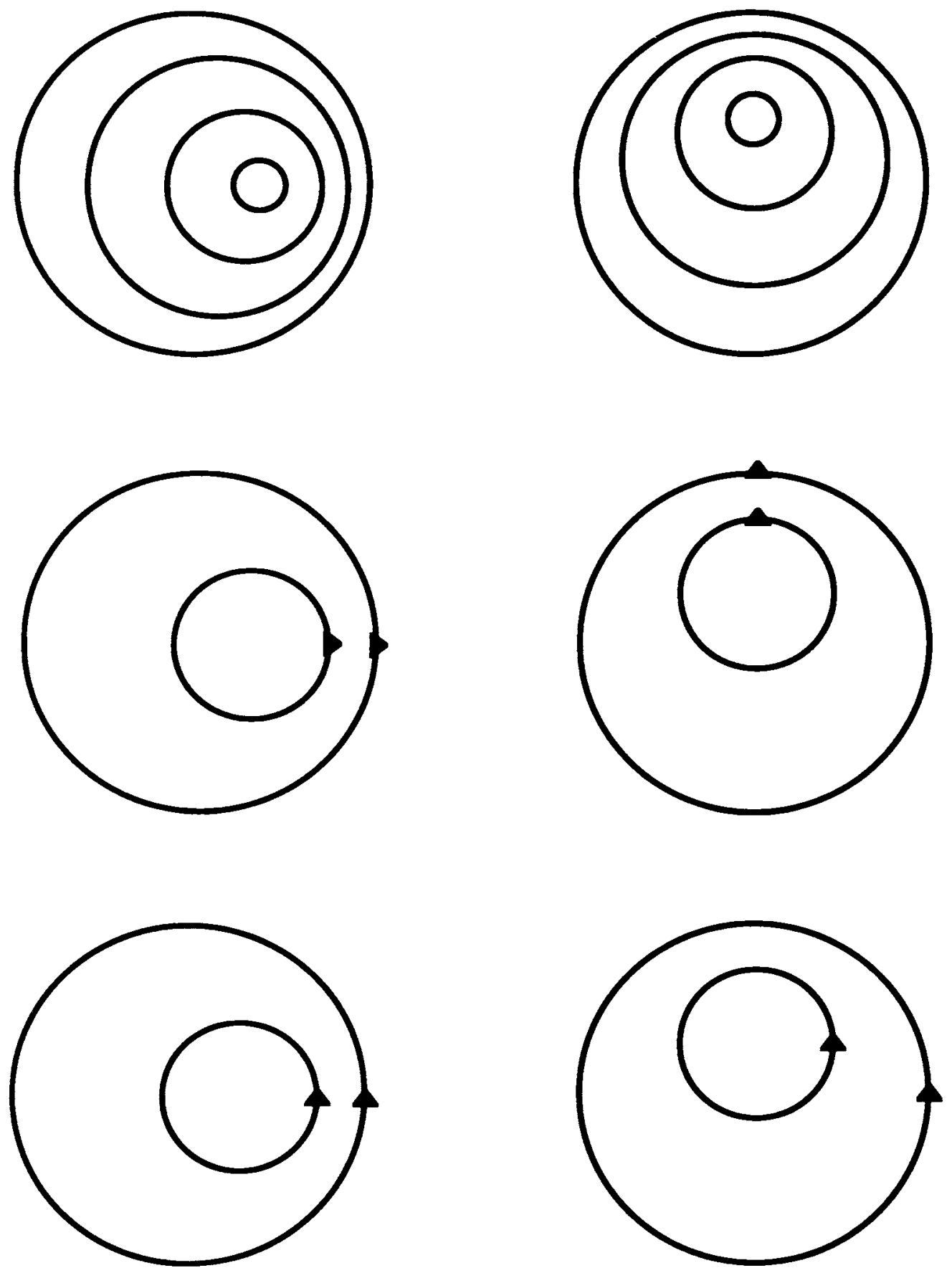

Figure 1. The top panel shows a traditional stereokinetic effect (SKE) cone display rotated $90^{\circ}$. The middle panel shows the change in position for two points on different contours. Here and in the bottom panel, only two contours are depicted. When an SKE pattern is perceived to have orientation stability, the apparent change in position for two points on different contours is as shown in the bottom panel.

lach, 1935; Wallach \& Centrella, 1990; Wallach, Weisz, \& Adams, 1956). When motion along the contours is not detected, the perceived pattern achieves what Musatti called orientation stability, and the contours appear to move relative to each other as is shown in the bottom panel of Figure 1. These contour-relative motions evoke the perception of a three-dimensional form, tilted in depth at an angle consistent with its eccentricity and apparent height and moving around the line of sight ( $z$-axis) with equal $x$ - and $y$-axis oscillatory components. This motion may best be appreciated using the following example. Hold a pencil by its base and point the tip toward you. Monocular viewing works best here. Tilt the major axis of the pencil slightly off of the line of sight so that the tip is at 3 o'clock relative to the base that will serve as a 
pivot. Now move the tip so as to draw a virtual circle in the picture plane while holding the base stationary. This is the motion seen for the major axis of the SKE cone.

\section{SKE and KDE Compared}

The KDE can be decomposed into two transformations, only one of which is present in the SKE. We first discuss this distinction in a manner specific to the SKE cone. Later a general account is provided.

Figure 2A shows two projections of contours on an actual shallow rigid cone separated by a $90^{\circ}$ rotation about the $z$ axis equivalent to that observed in the SKE cone depicted in Figure 2B. The two transformations of interest are as follows: (a) Between-contour motions are changes in orientation (and in situations to be discussed later, changes in projected distances) between contour centroids. For the KDE and SKE patterns depicted, the line connecting contour centroids rotates as would the hands of a clock from 3 to 12 o'clock. (b) Within-contour motions are the changes in projected distance that occur between any pair of points on the same contour as a result of the contour's changing observer-relative slant. For example, notice that because the patterns maintain phenomenal orientation, two points on a KDE contour at 3 and 9 o'clock change their relative projected distance as their contour changes its slant. KDE patterns manifest both transformations, whereas SKE patterns present only the betweencontour motions that are consistent with rigid-object rotation. The patterns in Figure $2 \mathrm{C}$ show only the within-contour motions. Here each of the contours deforms in a manner consistent with a planar surface changing slant; however, the distance between contour centroids remains constant; thus, the pattern is globally inconsistent with any rigid transformation. For convenience, we call this display the elastic effect (EE) because of its nonrigid appearance. (Experiment 1 documents the validity of this phenomenal description for EE.)

Two points need to be made. First, this definition of the stimulus basis for the SKE-between-contour motions that are consistent with small right-object rotations occurring in the absence of appropriate within-contour motions-allows for the creation of SKE displays that are not picture-plane rotations of two-dimensional patterns such as can be created on a turntable. Figure $3 \mathrm{~A}$ shows an SKE cone that is oscillating about the vertical axis. In this event the contours simply translate relative to each other. Figure $3 \mathrm{~B}$ shows three projections of an SKE square pyramid. In each projection, the locations of the inner squares' centers have been rotated counterclockwise by $45^{\circ}$ about the $z$-axis originating at the center of the largest square. Notice that orientation stability is specified by the fixed orientation of the square contours in this display rather than being the result of not detecting motion along circular contours as occurs in the SKE cone. The experiments to be reported here demonstrate that SKE displays created in these ways are perceptually equivalent to those that can be produced by picture-plane rotations of twodimensional patterns. We believe it is an interesting historical fact that, because of the technology available to them, Benussi and Musatti used a turntable to create the conditions for SKE. For them, it was necessary to use circular or nearly circular patterns in their displays so that orientation stability would occur and the contours would then appear to translate relative to one another. The translation is crucial. At present, using computer graphics animation, one can create the translation of contours easily; thus, it is no longer important to use circular contours on a turntable.

The second issue concerns the magnitude of the absent within-contour deformations and whether they are below perceptual threshold in SKE displays. A complete absence of these deformations is geometrically consistent with only an infinitely tall cone or infinitely deep funnel, and, of course, this is not what people observe. Consider the oscillating cone depicted in Figure 3A. For an actual rigid cone, the motion of the tip relative to the base is a function of the angle of oscillation and the height of the cone. Only an infinitesimal oscillation around the $y$-axis could yield no foreshortening of the projected contours in the picture plane; thus, the cone would have to be infinitely tall to project a changing eccentricity without contour foreshortening. However, the missing amount of contour foreshortening resulting from changing slant is just barely sufficient to deform the contours into ellipses that are noticeably different from circles. Shown to the right in Figure 4 is a rigid cone having a height and projected eccentricity equivalent to that perceived in the typical SKE cone to the left. (Perceived height is taken from assessments made in Experiment 6.) For this cone, the amount of slant needed to produce the projected eccentricity depicted is $14^{\circ}$, an orientation that results in a projected ellipse having a minor axis that is foreshortened by only $3 \%$. As Experiment 4 shows, this magnitude of within-contour deformation is detectable, but it is hardly salient. Thus, the SKE is perceived to have motions that are almost equivalent to those that would be produced by a rigid object moving in a manner consistent with the percept. The motions that occur between contours are equivalent to those that would be produced by a small rigid-object rotation. Appropriate withincontour motions are absent in the SKE; however, if they were present, their magnitude would be barely detectable. In essence, SKE patterns present motions that are almost equivalent to those observed when a rigid object undergoes small rotations in the neighborhood of $15^{\circ}$.

\section{Object-Relative Versus Observer-Relative Depth}

The most general way to define the two transformations that we have identified is as follows: Consider an object (e.g., a cone pointing toward an observer) in which the $z$-axis corresponds to the front-to-back principle axis of the object. For the cone, this is the symmetry axis from its tip to the center of its base. If the cone has contours painted on it, in planes parallel to its base, then distinct points on a single contour will not differ in their $z$ values but will be separated in the $x, y$ plane. Rotating this cone about the $x$-or $y$-axes, or both, produces motions between contours that are due to their separation in $z$-depth. It also produces within-contour motions that are due to the changing slant of the contour's $x$, $y$ planes. The SKE presents only the former motions: relative motions between points separated in $z$ but no changes for points with equivalent $z$ values. Thus, there are two motions that occur in small rotations of an object: those produced by differences in $z$-depth and those produced by changes in $x, y$ - 

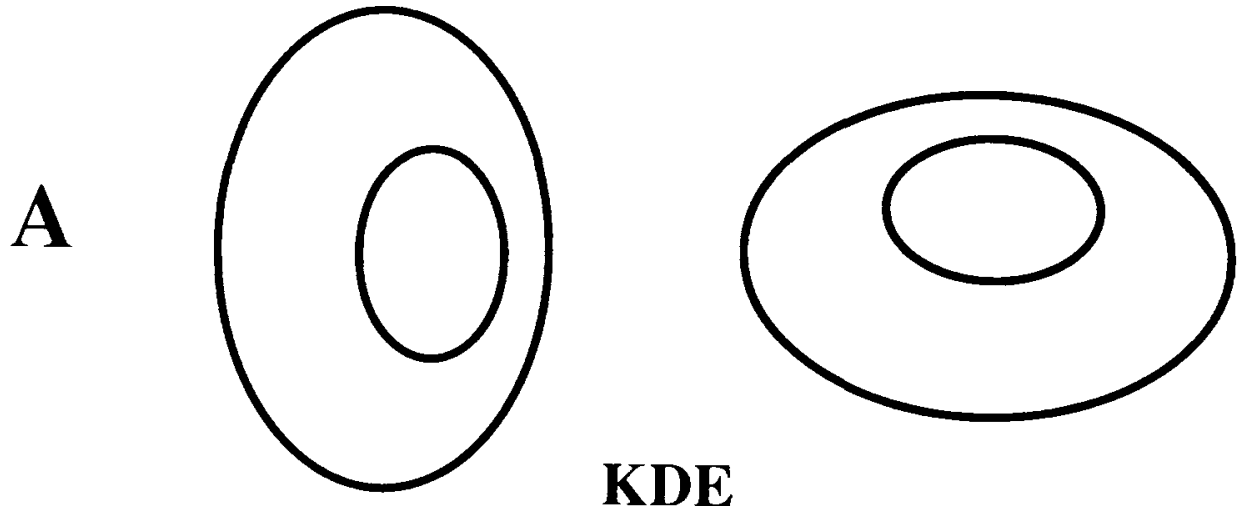

KDE

B
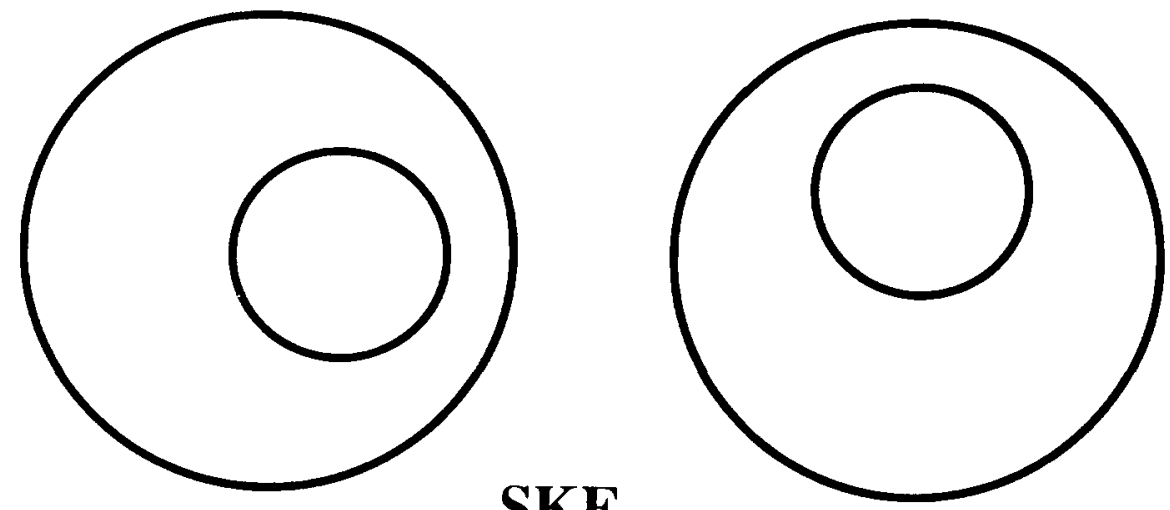

SKE
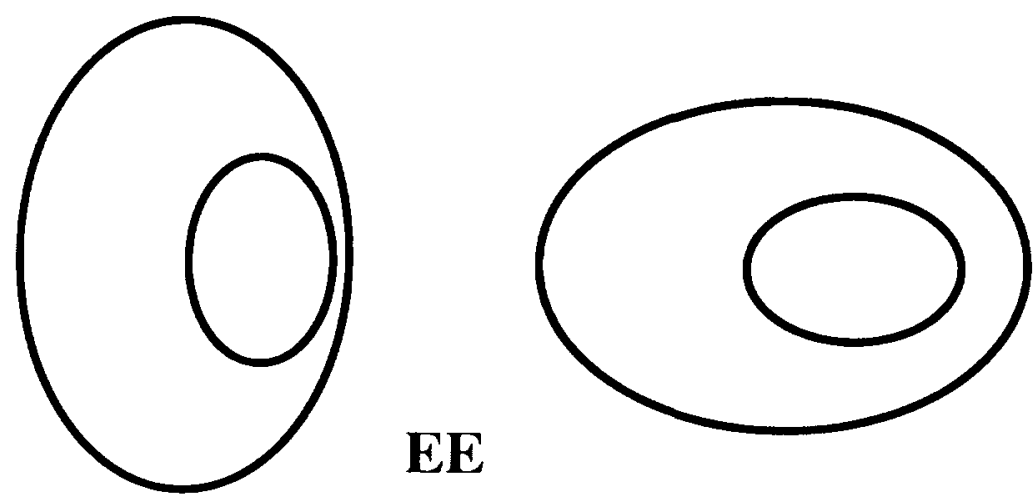

Figure 2. Panel A shows two contours on a rigid cone rotated $90^{\circ}$ so as to be consistent with the observed motions of a stereokinetic effect (SKE) cone. This kinetic depth effect (KDE) event can be decomposed into two distinct transformations. The first is depicted in Panel B and consists of betweencontour motions. here defined by a change in orientation of the contour centroids. (This transformation defines the stimulus basis for the SKE.) The second is shown in Panel $\mathrm{C}$ and consists of within-contour motions. (As shown, these contours change shape, but the distance and orientation of contour centroids remains unchanged. $E E=$ elastic effect.)

slant. The KDE manifests both; the SKE manifests only the former. In essence, the SKE exploits the transformations related to object depth while ignoring those within surfaces of the object that are incidental to changes in observer-relative slant. To reiterate, the object-relative motions that define the stimulus basis for the SKE are consistent with those that would occur in a small rotation of a rigid object; not any kind of object-relative motions will result in a depth percept; 

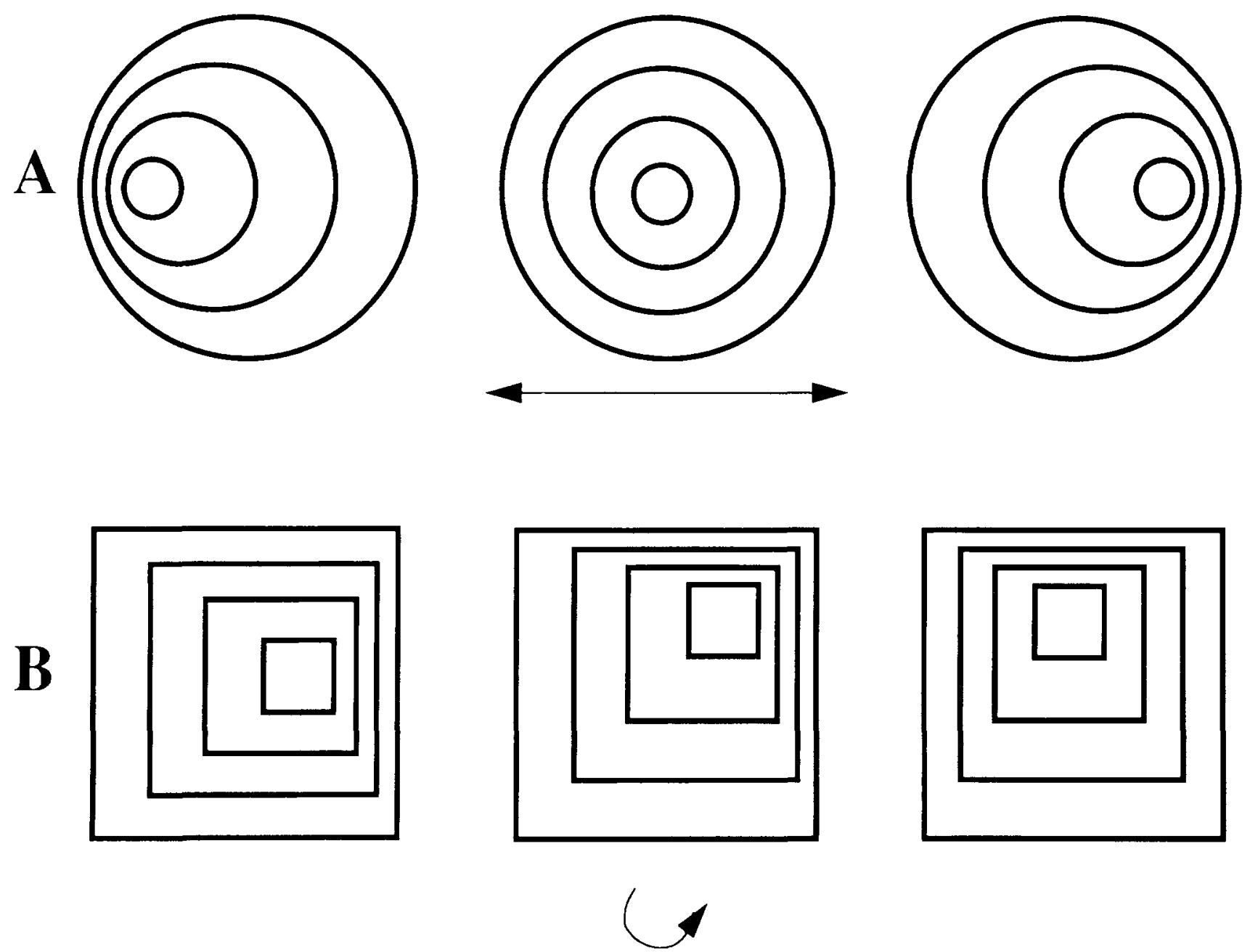

Figure 3. Panel A shows three images of a stereokinetic effect (SKE) cone oscillating about a vertical axis. Panel B depicts an SKE display consisting of nested squares in which the inner squares revolve within the outer contour while maintaining their orientation.

however, the appropriate observer-relative motions are absent.

\section{Overview to Experiments}

In the following experiments, we validate our definition of the SKE by showing that displays that are consistent with this definition but that cannot be produced by the method of rotating a two-dimensional pattern on a turntable are phenomenally equivalent to those that could be produced only by the traditional method. We also compare SKE stimuli with KDE stimuli manifesting salient within-contour transformations to determine whether the presence of these latter transformations results in the perception of more rigid or compelling depth impressions. It was found that the KDE and the SKE evoke equally compelling depth impressions and that rigidity differences favoring the $\mathrm{KDE}$ are slight. In addition, SKE displays in which contour rotations are apparent-using contours consisting of dots-are spontaneously perceived as three-dimensional objects with a latency that is not signifi- cantly greater than that for smooth contour displays. Finally, we created a random-dot SKE pattern consisting of a density gradient of dots that corresponded to the eccentricity of a typical SKE cone. The dots were assigned $x, y, z$ values according to their position on the virtual cone and moved according to the definition given previously; that is, only dots with different $z$ values moved relative to each other. The stimulus looks flat when static but pops into the appropriate three-dimensional form as soon as motion begins.

\section{Experiment 1}

In this experiment, we sought to determine whether the presence of highly salient within-contour transformations in KDE displays would result in more rigid or compelling depth impressions than are observed in matched SKE stimuli in which these transformations had been removed. In addition, we compared the traditional SKE cone with displays that appeared as triangular and square pyramids. As shown in Figure 3B for the square pyramid, these latter patterns pre- 


\section{S K E}

\section{K D E}
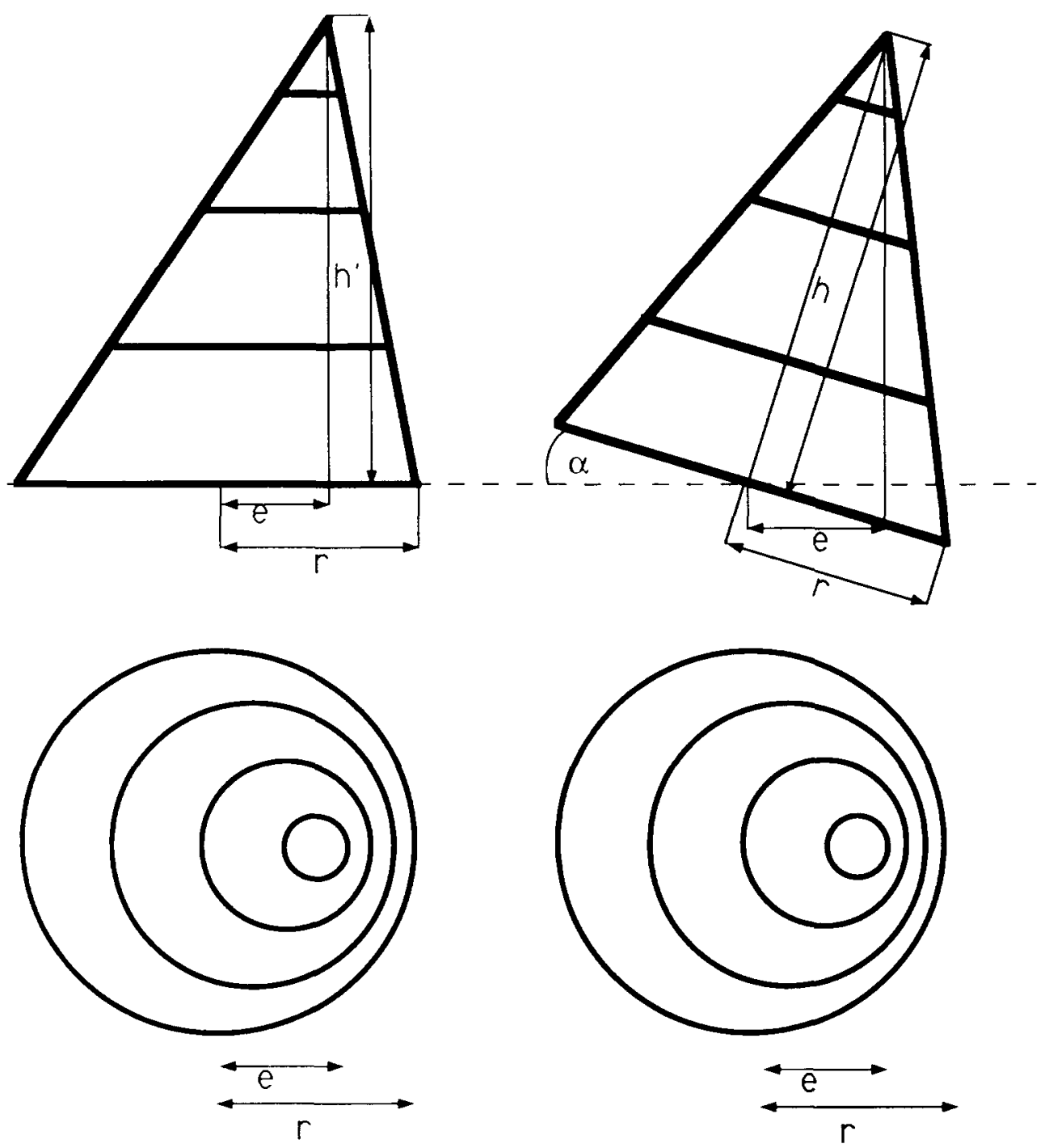

Figure 4. Schematics for stereokinetic effect (SKE) and kinetic depth effect (KDE) stimuli. (The top figures depict the side views of the virtual three-dimensional cones that project into the contours at the bottom. The virtual SKE and KDE objects are identical in radius of the base [r], eccentricity [e], and height, i.e., perceived height $\left[h^{\prime}\right]$ and objective height $[h]$ of SKE and KDE, respectively. The projections of these objects are identical except that the KDE contours are slightly foreshortened because of the virtual object's tilt $[\alpha]$.)

sented the apparent motions of the SKE cone-rotation with orientation stability-but they could not have been produced using the turntable method. We compared the traditional SKE cone to displays that were not simply rotations of twodimensional patterns to demonstrate their equivalence and to confirm our definition of the relevant SKE transformations.

\section{Method}

Subjects. Forty-eight University of Virginia undergraduates, 24 women and 24 men, were recruited from an introductory psychology course and received partial credit.

Stimuli. Figures 4 and 5 provide a general schematic for KDE and SKE stimuli, and the parameters defined there are referred to in 
this and subsequent experiments. The bottom panels depict the projected stimulus displays, and the top panels show the corresponding virtual three-dimensional objects. Typically, stimuli consisted of four nested contours. For KDE stimuli, the contours coincided with the projection of circles drawn on an unseen three-dimensional cone. These contour lines were parallel to the base of the cone and were equidistant from one another. The cone was tilted by a magnitude equal to $\alpha$ with respect to the picture plane; thus, the contours projected a nesting of eccentric ellipses (minor axis $=\mathrm{r} * \cos \alpha$ ). Stimulus eccentricity $(\mathrm{e} / \mathrm{r})$ was defined as the projected displacement of the cone's tip from the center of its base (e) over the radius of the base (r). Height (h) is the distance of the cone's base to its tip. For SKE stimuli, eccentricity is specified in the same manner as in KDE.
Within-contour changes are absent, and height is apparent $\left(h^{\prime}\right)$ and empirically determined.

Two new stimulus types were treated from the transformations inherent in the KDE. As is depicted in Figure $2 \mathrm{C}$, the first preserved within- but not between-contour motions. That is, the centers of the contours remained at a fixed distance and orientation relative to each other, but the contours themselves foreshortened sinusoidally just like those of a KDE stimulus. The motions of each contour were consistent with a projection of a rigid surface changing slant continuously: however. because the distance between contour centers did not change. the global motions were quite incompatible with those of any rigid object. Because of its nonrigid appearance, this stimulus was called $\mathrm{EE}$. The second new stimulus presented both the within-

\section{KDE}
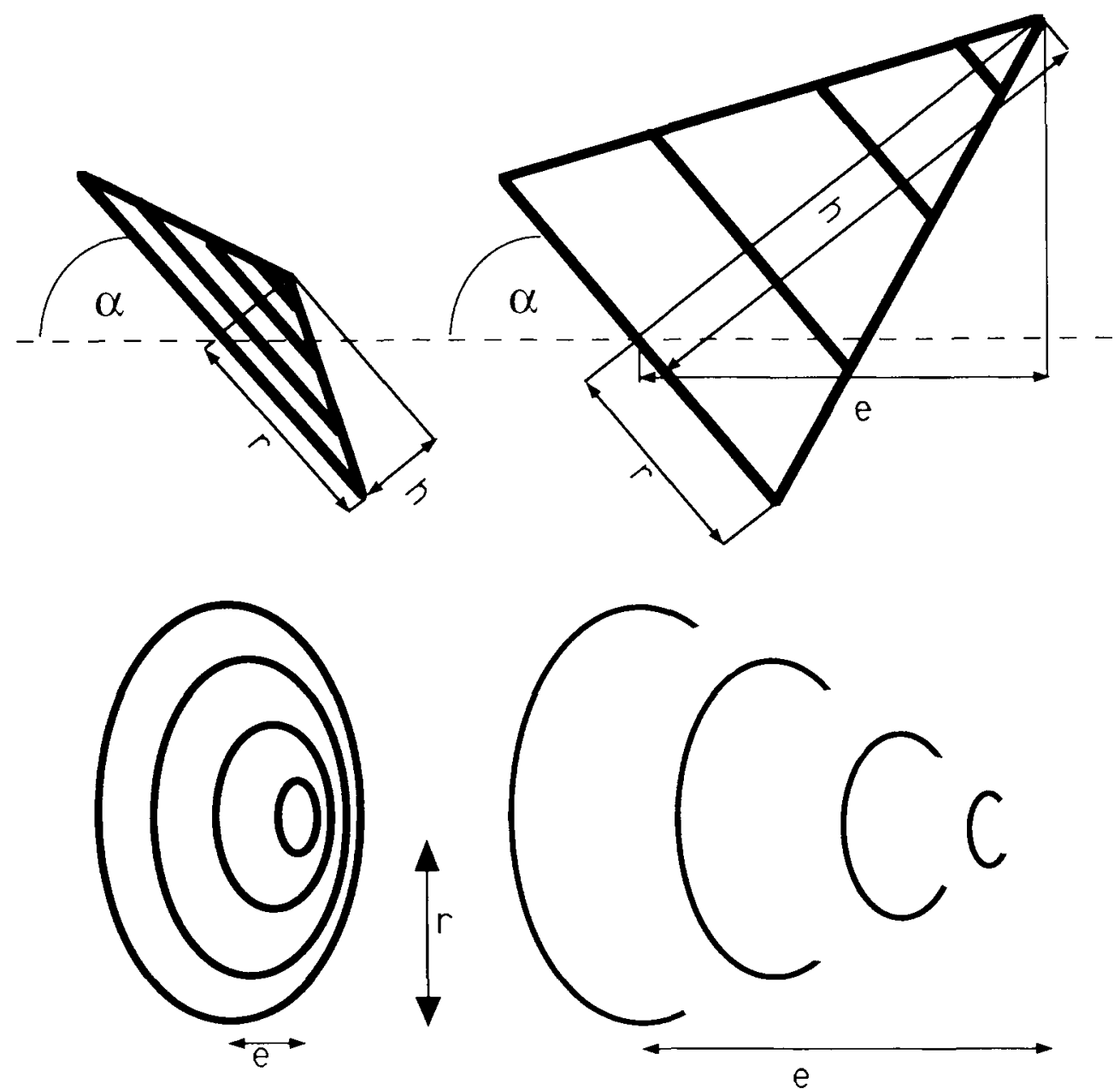

Figure 5. Schematics of two kinetic depth effect (KDE) stimuli differing in height (h) and eccentricity (e) but not in radius $(r)$. (For the stimulus on the left. $h=0.26 * 2 r$, whereas for the one on the right. $h$ $=1.25 * 2 \mathrm{r}$. Note that large eccentricity values cause the projected contours to be occluded as if the contours were drawn on an unseen opaque cone. $\alpha=$ tilt.) 
and the between-contour transformations of a KDE display: however, both of these motions were set at different phases within and across each contour. As with EE, the motions of each contour were consistent with a surface changing in projected slant: however, unlike EE. here each contour was always at a different instantaneous slant. Moreover, the between-contour motions were likewise out of phase; thus, for example, some contours moved to the left as others moved right. Again. this display was incompatible with any rigid-object interpretation, and we called it the chaotic effect (CE) because of its appearance. Both of these stimuli were created to serve as anchors for rating scales of depth and rigidity. CE in particular was of no theoretical interest.

Three stimulus shapes were used: One consisted of the traditional nested circles, one of nested equilateral triangular contours, and the third of nested squares. The KDE formats for the latter two shapes were triangular or square pyramids of the same height as the circular one. Their SKE formats had the same amount of between-contour motion as the circular SKE. Their contours did not change orientation; rather, their centroids received the same amount of $x$ - and $y$ axis motion that a turntable stimulus would have. Every stimulus consisted of four nested contours. Their bases were $2.0 \mathrm{~cm}$ (diameter for circles. length of base for squares and triangles). Rotation was at $0.65 \mathrm{~Hz}$. Moderate eccentricity settings $(0.4)$ were chosen for all stimuli to avoid overlap of the individual contours. KDEs, EEs, and CEs were foreshortened to $65 \%$ of the length of the major axis of the ellipses, which corresponded to a KDE slant of $49.5^{\circ}$. As is established in Experiment 4. this is far above threshold for the detection of such foreshortening. KDE height was $0.26 * 2 \mathrm{r}$.

A schematization of the KDE cone used in this experiment is shown to the left in Figure 5. Notice that to obtain a salient withincontour transformation, the cone's slant must be quite large. This slant, in conjunction with a typical SKE eccentricity value, dictates that the cone's height be relatively small. As is shown to the right in this figure. to match a KDE cone at this slant with the apparent height seen in the SKE. the cone's eccentricity must exceed 1.0; that is, the tip must extend beyond the base. and self-occlusion will occur. This latter KDE stimulus is compared with the SKE in Experiment 3 .

The stimuli were presented on a Sun $3 / 60$ workstation using a high-resolution graphics monitor $(33.5 \mathrm{~cm}$ wide $\times 25 \mathrm{~cm}$ high; 1.152 $\times 900$ pixels). Viewing distance was $64 \mathrm{~cm}$. A viewing box was used to facilitate the perception of phenomenal depth by reducing the conflicting cues indicating that the images were two-dimensional projections. The box was fitted over the screen, and its interior was painted black to reduce the reflection of light. Two windows inside the box occluded the borders of the computer screen and the inside walls of the viewing box. They were at about one quarter and one half of the total distance from the screen. At the observer's end, a scuba mask was mounted on the box to prevent light leakage. A slide with one viewing hole was inserted into the mask to ensure monocular viewing.

Design. A Latin-square design was used such that every stimulus was seen at each position in the series by exactly 4 subjects. That is, each observer saw one particular order of 12 stimuli ( 3 shapes and 4 categories, namely KDE, SKE. EE, and CE) and each stimulus was seen as the first one by exactly 4 observers.

Procedure. Stimuli were viewed monocularly in this and subsequent experiments. Observers could choose their preferred eye and switch eyes after the first presentation of all 12 stimuli if they wished. First, all stimuli were shown for $40 \mathrm{~s}$ each during which time observers reported their spontaneous descriptions of the stimulus, but they were given additional time if they wished. For every stimulus, subjects were asked to describe what they saw. Neutral follow-up questions were asked whenever the observer was hesitant to provide detailed information. The experimenter recorded the gist of the spontaneous report on paper, noting especially if the dimensionality of the stimulus (two dimensional vs. three dimensional) was mentioned or if a particular object was likened to the display.

A break was taken to introduce three rating scales (ranging from 1 to 10) for (a) amount of depth, (b) compellingness of depth, and (c) rigidity. Amount of depth was explained in terms of how far the object would stick out or recede into the screen; ratings could range from flat to very pronounced. Subjects were told that they had already seen all stimuli during the first part of the experiment, and they were asked to apply the rating scale such that the most shallow stimuli would get low ratings and the deepest ones. high ratings. Compellingness was explained as the sense of three dimensionality and "realness" that the observer would get from the stimulus. The example of a photograph of an object (less compelling) and the object itself (more compelling) was used to emphasize that compellingness should be a separate dimension from amount of depth. When explaining rigidity, observers were alerted to the fact that motion should not be confounded with elasticity. An arbitrary moving object (e.g., airplane) was given as an example for a rigid object and jello or a rubber band for an elastic one. Use of the whole scale based on the range of stimuli seen in the first phase of the experiment was encouraged for all three rating scales to anchor them as well as possible. Then the stimuli were presented in the same order again, and subjects made their ratings by verbally reporting the value selected to the experimenter. It usually took observers about $15 \mathrm{~s}$ per stimulus. but they were told to take as much time as they needed.

\section{Results and Discussion}

Spontaneous reports were coded as to whether three-dimensional objects were mentioned. Both SKE and KDE displays evoked spontaneous mentioning of such objects from the vast majority of observers, whereas such objects were less often mentioned for EE and CE stimuli. For $79.2 \%$ of all KDE displays and $84.0 \%$ of all SKE displays, three-dimensional objects were spontaneously mentioned. Typically, the KDE displays were referred to as frisbees or hub caps, whereas the SKE displays were referred to as cones, tunnels. or pyramids. Only $39.6 \%$ of the EE displays and $24.3 \%$ of the CE displays evoked the report of objects. Among those, EEs were typically referred to as jello and CEs as a solar system.

With regard to the rating scales, no position effects were found. They will, therefore, not be considered or analyzed in the following account. The data for the three rating scales are shown in Figure 6. In general, SKE and KDE were rated considerably higher on depth, compellingness, and rigidity than CE and EE. More specifically, the findings for each scale were as follows:

Depth. A repeated measures analysis of variance (ANOVA) revealed a main effect for shape, $F(2,92)=8.05, p<$ .001 . Stimuli made up of circles were judged as deeper than those consisting of triangles, $F(1,46)=8.49, p<.006$, and squares, $F(1,46)=11.6 . p<.002$. Triangles and squares did not differ significantly $(p>.05)$. There was a main effect for category of stimulus, $F(3,138)=113.84, p<.0001$. SKE was judged as deeper than KDE, $F(1,46)=134.07, p<.0001$. EE. $F(1,46)=305.19, p<.0001$, and CE. $F(1,46)=175.29$. $p<.0001$. KDE was judged as deeper than EE, $F(1.46)=$ 71.72. $p<.0001$, and $\mathrm{CE}, F(1,46)=20.18, p<.0001$. EE and $C E$ did not differ significantly. 
Amount of Depth

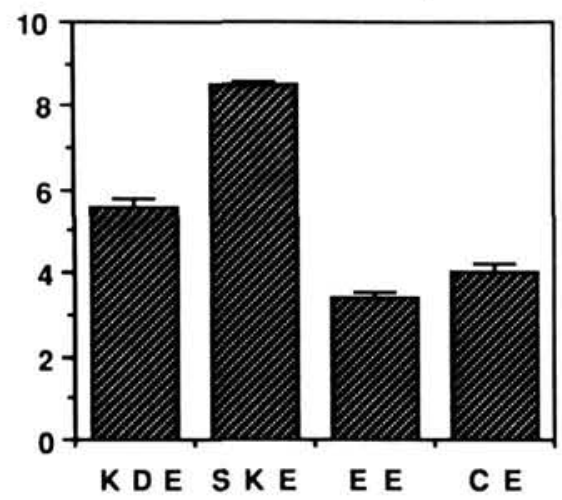

Depth Compellingness

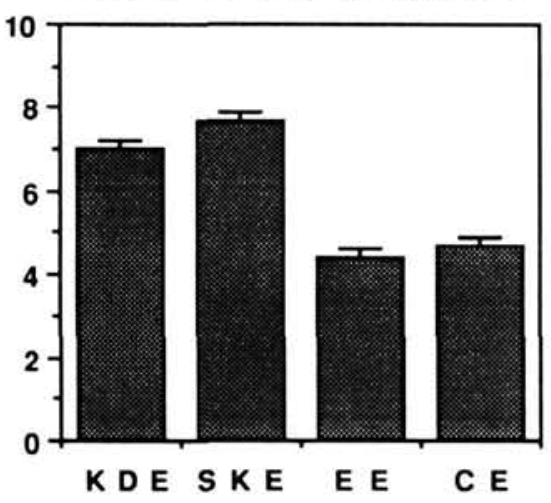

Rigidity

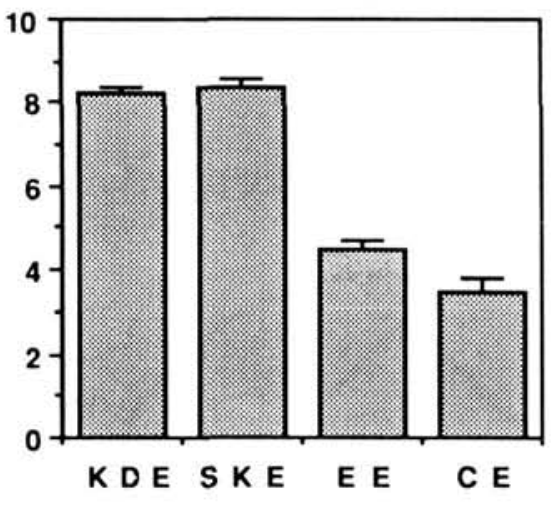

Figure 6. The mean ratings for amount of depth, depth compellingness, and rigidity for each stimulus category in Experiment 1. (The rating scales ranged from 1 [minimum] to 10 [maximum]. $\mathrm{KDE}=$ kinetic depth effect; SKE = stereokinetic effect; $E E=$ elastic effect; $\mathrm{CE}=$ chaotic effect.)

Compellingness. No effect for shape, $F(2,92)=0.56, p>$ .5 was found, but category of stimulus had a significant influence, $F(3,138)=43.71, p<.0001$. SKEs looked more compelling than KDEs, $F(1,46)=6.62, p<.014$, EEs, $F(1$, $46)=119.69, p<.0001$, and CEs, $F(1,46)=54.03, p<$ .0001 . KDEs were judged as more compelling than EEs, $F(1$,
$46)=85.83, p<.0001$, and CEs, $F(1,46)=25.34, p<.0001$. EEs and CEs were not significantly different.

Rigidity. A main effect was found for category of stimulus, $F(3,138)=73.98, p<.0001$, but not for shape, $F(2,92)$ $=2.52, p>.08$. SKEs were judged more rigid than EEs, $F(1$, $46)=88.07, p<.0001$, and CEs, $F(1,46)=148.19, p<$ .0001 . KDEs were rated more rigid than EEs $F(1,46)=$ $104.34, p<.0001$, and CEs, $F(1,46)=95.1, p<.0001$. SKEs and KDEs did not differ significantly; EEs and CEs did differ slightly, $F(1,46)=4.29, p<.05$.

Summary. SKE and KDE stimuli were quite similar in their appearance as three-dimensional objects. They received equivalent judgments on rigidity, and SKEs were rated as being slightly more compelling. As expected, given the small simulated height in the KDE displays, these stimuli received lower depth ratings relative to SKE. These data support the conclusion that the presence of salient within-contour transformations in $\mathrm{KDE}$ do not result in more rigid or compelling depth percepts relative to matched SKE patterns. Moreover, the results for the triangular and square contour SKE displays indicate that they evoked three-dimensional percepts that are similar to those seen in the traditional SKE cone. Thus, we see that the SKE depends not on the picture-plane rotation of a two-dimensional pattern but rather on the presence of appropriate between-contour transformations.

As to the relatively poor perception of depth, compellingness, and rigidity found for the EE displays in which only within-contour transformations occurred, it should be noted that within-contour deformations alone can be effective in creating an impression of three-dimensionality when there is only a single contour. Thus, in their article on KDE, Wallach and O'Connell (1953) showed that a deforming line (e.g., the projection of an oblique rod rotating about a vertical axis) will yield the impression of a rigid rod rotating in depth. From this and other examples, they concluded that the stimulus conditions necessary for the KDE include change of both length and orientation of the contour, a conclusion that has been widely accepted by investigators. Apparently, then, the result for our EE displays is based on the fact that the absence of between-contour transformations is inconsistent with the presence of within-contour transformations.

In single-contour cases in which good depth perception occurs, we are not talking about transformations that lead to the impression of a three-dimensional object so much as to the impression of a two-dimensional contour or surface changing in orientation with respect to the observer. In the experiments described here, by using more than single-contour displays, we are talking about the creation of or failure to create impressions of three-dimensional objects undergoing rotation.

\section{Experiment 2}

This study replicated the previous design and introduced three new stimulus shapes: an elliptical cone and two patterns in which the nested contours did not conform to familiar or regular objects. Especially with regard to the latter two stimuli, we sought to determine whether the SKE transformation would result in compelling, rigid, three-dimensional percep- 
tions for contours that did not coincide with those of simple regular objects.

\section{Method}

Subjects. Twelve University of Virginia undergraduates, 6 women and 6 men, were recruited from an introductory psychology course and received partial credit. None of them had participated in previous SKE experiments.

Stimuli. Three new stimulus shapes were introduced. First, SKE stimuli were created, the contours of which were elliptical such that their aspect ratio matched the foreshortening of the KDE cone used in this experiment and in the previous one. These SKE contours maintained orientation stability; the major axes of the ellipsis were always oriented vertically. Parameters for the KDE stimulus were: $\alpha$ $=49.5^{\circ}, \mathrm{e} / \mathrm{r}=0.4, \mathrm{~h}=0.26 * 2 \mathrm{r}$. This tilt led to $65 \%$ foreshortening of its minor axis, and the elliptical SKE also had an aspect ratio of .65 . The diameter of the major axis was $2 \mathrm{~cm}$ for all stimuli. The SKE also had an eccentricity of 0.4 . Second, regularly nested and randomly nested polygons were created, and these are depicted in Figure 7. For the regularly nested polygons, the number of sides for each contour decreased as a function of size: The largest contour (base) had 8 sides and the smallest, 4 . For random polygons, each contour was assigned a random number of sides between 16 and 4 (with the constraint that only the largest shape could have 16 sides to avoid overlap of contours without reducing eccentricity below 0.4 ). The stimulus parameters for polygons were identical to the elliptical stimuli except for shape. EE and CE analogues were created, resulting in a set of 12 different stimuli (KDE, SKE, EE, and CE for ellipses, regularly nested, and randomly nested polygons). Rotation for all stimuli was $0.65 \mathrm{~Hz}$. Viewing distance and conditions were equivalent to those of Experiment 1.
Design and procedure. The same Latin-square design was used as in Experiment 1. Stimuli were presented once to obtain spontaneous reports and once for ratings of amount of depth, compellingness, and rigidity.

\section{Results and Discussion}

The percentages for cases in which observers reported threedimensional objects (cones, tunnels, frisbees, and so on) were distributed as follows: $\mathbf{7 9 . 2 \%}$ for ellipses, $54.2 \%$ for regularly nested polygons, and $50.0 \%$ for randomly nested polygons; $86.2 \%$ for SKEs, $58.3 \%$ for KDEs, $52.8 \%$ for EEs, and $47.3 \%$ for CEs. Elliptical SKEs received the highest number of reported three-dimensional objects $(91.7 \%)$.

The ratings showed no significant effect for stimulus shape on KDEs. For SKEs however, elliptical shapes were slightly more compelling than other shapes, but they had the same amount of depth as polygons. Unlike the previous experiment, here SKEs and KDEs did not differ in compellingness. Again the depth difference found for SKE and KDE stimuli was expected because of the small height simulated in the KDE displays.

Depth. The mean ratings were 7.5 (SKE), 2.9 (KDE), 3.2 (EE), and 5.2 (CE); 5 (ellipses), 4.7 (nested polygons), and 4.4 (random polygons). A repeated measures ANOVA revealed no main effect for shape, $F(2,18)=.59, p>.5$, but a main effect for category of stimulus, $F(3,27)=21.02, p<.0001$. SKEs were judged deeper than KDEs, $F(1,9)=74.88, p<$ .0001 , EEs $F(1,9)=69.72, p<.0001$, and CEs, $F(1,9)=$ $22.07, p<.0001$. KDEs were judged as deeper than CEs, $F(1$,

\section{Nested Polygons}

\section{Regularly Nested}

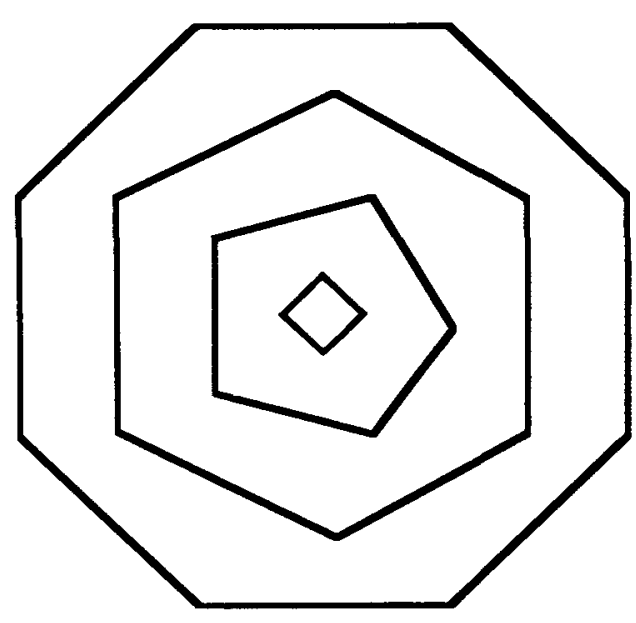

\section{Randomly Nested}

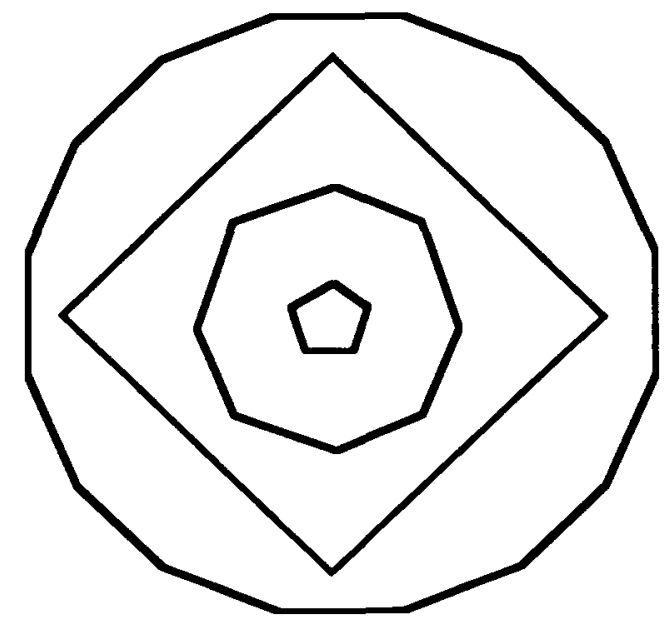

Figure 7. Exemplars of nested polygon stimuli. (The stimulus on the left consists of regularly nested polygons in which number of sides decreases progressively toward the center. The stimulus on the right consists of randomly nested polygons in which number of sides is independent of their closeness to the center.) 
$9)=6.32, p<.04$. CEs were rated slightly deeper than EEs, $F(1,9)=5.24, p<.05$.

Compellingness. The mean ratings were 6.5 (SKE), 5.9 (KDE), 4.8 (EE), and 4.9 (CE); 6.6 (ellipses), 5.1 (regularly nested polygons), and 4.8 (randomly nested polygons). A main effect for shape, $F(2,18)=4.07, p<.04$, was found: Ellipses looked marginally more compelling than regularly nested polygons, $F(1,9)=4.68, p<.06$, and randomly nested polygons, $F(1,9)=4.61, p<.06$. A main effect for category of stimulus, $F(3,27)=3.74, p<.03$, revealed that SKEs do not differ from KDEs, but they looked more compelling than EEs, $F(1,9)=23.95, p<.0009$, and CEs, $F(1,9)=5.51, p$ $<.05$. Elliptical SKEs look slightly more compelling than polygon SKEs or KDEs, $F(1,9)=7.6, p<.03$. The interaction of category of stimulus and shape was not significant, $F(6$, 54) $=1.6, p>.15$.

Rigidity. The mean ratings were 6.9 (SKE), 7.2 (KDE), $4.2(\mathrm{EE})$, and 4.8 (CE); 6.3 (ellipses), 5.5 (regularly nested polygons), and 5.5 (randomly nested polygons). Shape had no significant effects, $F(2,18)=2.76, p>.1$, but category of stimulus did, $F(3,27)=5.65, p<.004$. SKEs were judged more rigid than EEs, $F(1,9)=19.38, p<.002$, as were KDEs, $F(1,9)=8.97, p<.02$.

Summary. The pattern of results here was essentially the same as those of the previous experiment. We conclude that the SKE transformation need not be applied to simple regular contour nestings in order for rigid, compelling depth impressions to be evoked.

There were two follow-up studies of Experiment 2 that we describe only briefly here. First, we sought to obtain spontaneous reports for the irregularly nested contour displays because in the previous study the reported perception of threedimensional objects may have been primed by having observed similar objects in the more regular displays. Here each subject only saw one stimulus.

Forty observers were shown either an SKE or a KDE version of a circular cone or of a randomly nested polygon display. Thus, each unique stimulus was shown to 10 different observers. Spontaneous reports were obtained, and the results revealed that both circular stimuli evoked impressions of three-dimensional objects $100 \%$ of the time, whereas random polygon KDEs and SKEs were associated with three-dimensional objects $80 \%$ and $70 \%$ of the trials, respectively. These findings suggest that the SKE transformation is about as likely to evoke definite three-dimensional percepts for randomly nested polygons as are $\mathrm{KDE}$ motions.

In a second follow-up study, we assessed perceived height for $\mathrm{KDE}$ and SKE versions of three different stimulus shapes (circular cones, regularly nested polygons, and randomly nested polygons) by measurement rather than by ratings. The precise method for measuring perceived height is described in Experiment 6 . The result was that the SKE transformation evoked equivalent apparent heights for the three patterns having quite different contours but the same eccentricity.

\section{Experiment 3}

In Experiments 1 and 2, we introduced salient withincontour transformations to determine whether they would evoke more compelling, rigid depth impressions than are observed in SKE displays that lack these motions. It was found that KDEs so constructed did not differ from their matched SKE versions. However, in these experiments, the simulated height of the KDE stimuli was quite a bit smaller than what is perceived in the SKE displays, and that fact might have influenced the results. For example, the greater height in the SKE displays may have affected compellingness ratings even though these ratings were supposed to have been made independent of amount of depth.

In this experiment, we replicated Experiments 1 and 2 with $\mathrm{KDE}$ and SKE stimuli that were matched in phenomenal height. To create $\mathrm{KDE}$ displays having salient within-contour transformations and the appropriate height, the tips of their virtual objects must be projected beyond their base as is shown to the right in Figure 5. Thus, all stimuli, KDE and SKE, were given eccentricity values greater than $1(e>r)$, and contours were programmed to simulate partial occlusion as if by the objects' opaque but unseen form.

\section{Method}

Subjects. Twelve University of Virginia undergraduates, 6 women and 6 men, were recruited from an introductory psychology course and received partial credit. None of them had participated in previous SKE experiments.

Stimuli. As in the previous experiments, KDE, SKE, EE, and CE stimuli were used; in this experiment they had elliptical, rectangular, and regularly nested polygon contours. However, KDE and SKE displays were matched in perceived height using a procedure similar to that described in detail in Experiment 6 . Depicted to the right in Figure 5, a KDE stimulus was picked $\left(\mathrm{h}=1.25 * 2 \mathrm{r}, \alpha=49.5^{\circ}\right.$, foreshortening $=35 \%, \mathrm{e} / \mathrm{r}=1.9$ ) that ensured overlap of its contours. The SKE stimuli were chosen such that their eccentricity values $(e / r$ $=1.0)$ produced comparable perceived heights. For these SKEs, e was set to be equal to the major axis radius of the base; thus, the display's elliptical contours would just touch when their major axes coincided but would overlap everywhere else. Corresponding EE and $\mathrm{CE}$ versions of the stimulus were created. The base of all elliptical stimuli was about $1.5 \mathrm{~cm}$ wide and $2.3 \mathrm{~cm}$ long. Rotation was 0.65 $\mathrm{Hz}$. All stimuli had some overlap of contours as a result of large eccentricity and the choice of motion parameters. Rectangles and polygons had the same size, aspect ratios, and motion parameters. In the graphics displays, those (hidden) lines that were overlapped by smaller contours were removed such that the stimuli would selfocclude in the manner of projections of real objects (at least for the KDE cases).

Design and procedure. The same Latin-square design as in Experiment 1 was used; every stimulus was seen once per subject resulting in a total of 144 observations ( 3 shapes, 4 stimulus categories, and 12 subjects). The stimuli were presented twice: once for spontaneous reports and once to obtain ratings $(1-10)$ of amount of depth, compellingness, and rigidity.

\section{Results and Discussion}

SKEs and KDEs did not differ in terms of spontaneous reports of ratings of amount of depth and rigidity. SKEs were judged to be more compelling than KDEs, and ellipses were found to be more compelling than polygon stimuli.

Spontaneous reports showed that elliptical stimuli evoked mentioning of objects $83.3 \%$ (KDE) and $100 \%$ (SKE) of the 
time, whereas polygons did so $41.7 \%$ (KDE) and $58.3 \%$ (SKE) of the time. Rectangles were associated with objects $41.7 \%$ (KDE) and $50 \%$ (SKE) of the time.

The means for the rating scores are presented in Figure 8. The specific effects are now described.

Depth. No effect was found for shape, $F(2,20)=0.59, p$ $>.5$, but one was found for category of stimulus, $F(3,30)=$ $9.99, p<.0001$. SKE was judged as deeper than EE, $F(1,10)$

\section{Amount of Depth}

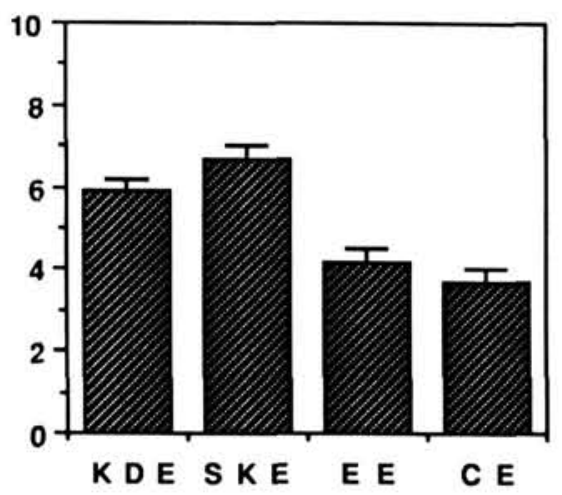

\section{Depth Compellingness}

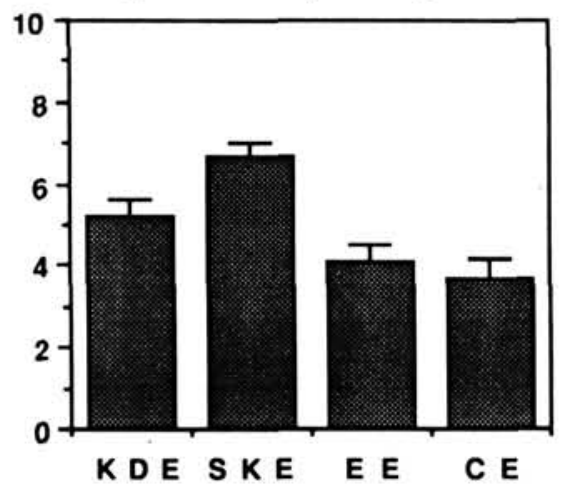

Rigidity

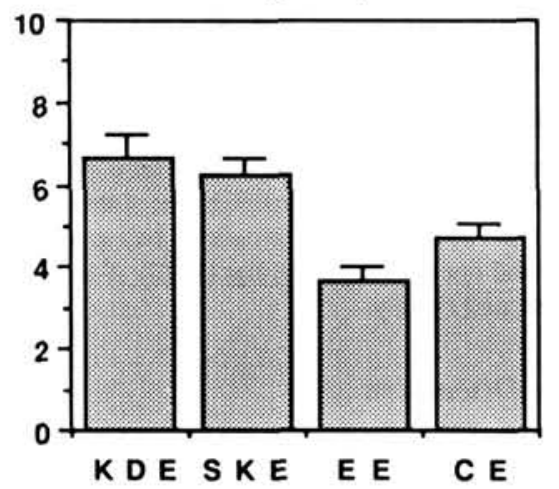

Figure 8. The mean ratings for amount of depth, depth compellingness, and rigidity for each stimulus category in Experiment 3. (The rating scales ranged from 1 [minimum] to 10 [maximum] $. \mathrm{KDE}=$ kinetic depth effect; SKE = stereokinetic effect; EE = elastic effect; $\mathrm{CE}=$ chaotic effect.)
$=17.40, p<.002$, and CE, $F(1,10)=32.55, p<.0002$. KDE was judged as deeper than EE, $F(1,10)=8.0, p<.02$, and $\mathrm{CE}, F(1,10)=7.44, p<.03$. Neither $\mathrm{KDE}$ and $\mathrm{SKE}$ nor EE and $\mathrm{CE}$ differed significantly.

Compellingness. A main effect for shape, $F(2,20)=6.15$, $p<.009$, revealed that ellipses looked more compelling than polygons, $F(1,10)=14.76, p<.004$. A main effect for category of stimulus, $F(3,30)=7.23, p<.0009$, was found: SKEs looked more compelling than KDEs, $F(1,10)=11.52$, $p<.007$, EEs, $F(1,10)=15.59, p<.003$, and CEs, $F(1,10)$ $=14.11, p<.004$.

Rigidity. No effects were found for shape, $F(2,20)=1.21$, $p>.3$, but a main effect was found for category of stimulus, $F(3,30)=6.62, p<.002$. SKEs were judged as more rigid than EEs, $F(1,10)=14.89, p<.004$. KDEs were rated as more rigid than EEs, $F(1,10)=38.35, p<.0001$. CEs did not differ significantly from any other condition.

\section{Experiment 4}

In an experiment that we do describe here in detail, we established the discriminability of SKE and KDE cone-like stimuli matched in all respects except that the within-contour transformations present in KDE were removed in the SKE version of this event. Musatti (1928-1929) argued that to exceed the discriminability threshold between circles and ellipses a rigid cone of a height equivalent to that observed in an SKE cone would have to be tilted by $20^{\circ}$ or more. However, we were able to demonstrate that observers can discriminate between KDE and SKE cones when they see them side by side even if the within-contour transformation is as little as $3 \%$ for the KDE stimulus. Depicted in Figure 4, this value corresponds to a $14^{\circ}$ tilt of a cone having an objective height and motion equivalent to that perceived in the SKE cone.

Therefore, although we were able to demonstrate this discriminability between SKE and KDE displays, presumably on the basis of the absence of within-contour transformations in SKE, our other experiments failed to show any difference in rigidity between these two kinds of displays. This is somewhat of a contradiction. It seemed to us that SKE stimuli had a very subtle nonrigid appearance that was not observed in $\mathrm{KDE}$ as would be expected given the absence of withincontour change. In this experiment, a more sensitive measure for rigidity was done using paired comparisons. KDEs, SKEs, EEs, and CEs for different shapes were compared directly in a forced-choice paradigm.

\section{Method}

Subjects. Twelve University of Virginia undergraduates, 6 women and 6 men, were recruited from an introductory psychology course and received partial credit. None of them had participated in previous SKE experiments.

Stimuli. KDE, SKE, EE, and CE stimuli that were of the same approximate perceived height were created in three different shapes (ellipses, randomly nested polygons, and rectangles) resulting in 12 unique stimuli. The stimulus parameters were identical to those used in Experiment 3 (i.e., all displays had overlapping contours and, thus, 
self-occlusion). Two different stimuli were displayed side by side. Both rotated at $0.75 \mathrm{~Hz}$.

Design. Forced-choice planned comparisons were made. Each of the 12 stimuli was compared with every other stimulus. leading to a total of 66 comparisons per subject. For a given stimulus pair, it was randomly determined which stimulus would appear on the left or the right side of the computer screen.

Procedure. Observers were tested individually. After the concept of rigidity had been explained (emphasizing that motion is independent from rigidity), subjects were asked to use the mouse to move a marker below the stimulus that looked more rigid. Four practice trials using stimuli not contained in the set of test stimuli were given to ensure that observers followed the instructions.

\section{Results and Discussion}

Our personal observations were confirmed: KDE was judged to be more rigid than SKE most of the time. Frequencies of preferences were as follows: KDE and SKE were strongly preferred over EE and CE (SKE over EE in $83.3 \%$ of the comparisons and SKE over CE in $74.1 \%$; KDE over $\mathrm{EE}$ in $86.1 \%$ and $\mathrm{KDE}$ over $\mathrm{CE}$ in $90.7 \%$ ). EE was preferred slightly over $\mathrm{CE}(61.1 \%)$. KDE was preferred over SKE $(76.9 \%)$. A similar picture emerges when we compared according to subject which stimulus was preferred for a given comparison: 11 of 12 subjects selected KDEs more often than EEs. For SKE versus EE, this ratio was 10:12; for KDE versus SKE, it was 10:12. This pattern did not change with stimulus shape.

\section{Experiment 5}

As discussed early in this article, the orientation stability observed in the traditional SKE seems likely to be due to an inability to detect motion in the direction of the smooth circular contour. Thus, one would surmise that if the contours' rotational motions were detectable, perhaps by having them consist of dotted lines, then the two-dimensional percept of a rotating pattern would dominate. As improbable as it seems, Musatti (1975) reported that a three-dimensional cone is still seen in such a case. He constructed a display using dashed lines for contours and reported the occurrence of stereokinesis in which the dashed lines were observed to rotate about a virtual cone that maintained orientation stability. Rock (1983) argued that orientational stability was a necessary precondition for the SKE and reported that circles consisting of dotted contours did not yield an SKE in his laboratory.

The present study was designed to assess more carefully Musatti's observation. A one-trial procedure was used to gain some clarity about phenomenal impressions of SKE displays that consisted of dotted circular contours. Latency to mention a three-dimensional percept was the primary dependent measure.

\section{Method}

Subjects. Twenty-four University of Virginia undergraduates, 12 women and 12 men, were recruited from an introductory psychology course and received partial credit. None of them had participated in SKE experiments before.

Stimuli. A circular standard SKE cone was used as a control stimulus: its base subtended $2.3 \mathrm{~cm}$. eccentricity was 0.63 , and it consisted of five circles. The motion corresponded to that of a turntable stimulus for which the center of its base was displaced in the direction of its tip by 0.5 radii from the turntable center. An identical stimulus was created that had dotted contours. The largest circle consisted of 42 evenly spaced dots whose diameters were 0.5 $\mathrm{mm}$. The smaller circles consisted of proportionately fewer dots. Rotation rate was $0.32 \mathrm{~Hz}$ for both stimuli.

Design and procedure. A between-subjects design was used in which half of the subjects viewed the control stimulus and half viewed the one with the dotted contours. Subjects were tested individually in a dimly lit room. Monocular viewing with the viewing hood was used. Before viewing the display, observers were instructed that one of the following phenomena could occur: The stimulus could initially appear to be flat and stay flat: it could seem to start out flat and then turn three-dimensional; it could start as a three-dimensional percept and stay that way or turn flat. The task was to report spontaneously what they saw but mention if the stimulus appeared to reorganize. The time that it took the subjects to report three-dimensionality was measured. The display was viewed until clear mention of a threedimensional object had been made or until $1 \mathrm{~min}$ had elapsed. During that time. spontaneous reports were recorded.

\section{Results and Discussion}

No significant differences existed between continuous and dotted contours for the time it took to report a three-dimensional percept. It took marginally longer before observers reported a stimulus organization in three dimensions for dotted contours: $13.4 \mathrm{~s}$ on average as opposed to $5.1 \mathrm{~s}$ for continuous contours, $F(2,23)=2.6, p>.1$. (Although this difference in means is relatively substantial, it is due largely to 1 subject who never saw the dotted contour display as three-dimensional, and her time was scored as $60 \mathrm{~s}$. Removing this subject's data reduces the mean for the dotted-contour display to 9 s.) In agreement with Musatti's findings, our observers described the dotted-line display as a contour that maintained orientation stability while the dotted lines revolved around it.

Two possible hypotheses for this finding come to mind. First. it may be that the dotted lines were blurred by their motion into smooth contours in the low spatial frequency channels and that the depth percept was driven by the motions derived from these channels. Alternatively, relevant betweencontour motions may be segregated by processes of perceptual organization. That is, the rotation along the contour is noticed. but the between-contour motions are perceptually specified by the relative motions of the contour centroids. Further research is needed here. Interestingly, orientational stability does occur for the virtual cone, possibly attesting to this crucial step in the process, and the motion of the dots is resolved as a rotation around this cone.

\section{Experiment 6}

In this study, we compared the apparent height of traditional SKE cones-picture-plane rotations of eccentric circles-with displays that conform to our definition of the SKE transformation but that could not be produced by this turntable methodology. The traditional SKE cone projects equal $x$ - and $y$-axis sinusoidal motions. Two new events were cre- 
ated that were in all respects the same as the traditional cone except that the vertical motion component either was reduced by one half or was absent altogether. This latter event is shown in Figure $3 \mathrm{~A}$. We found that our own viewing of these three patterns evoked equivalent percepts of virtual three-dimensional cones. A procedure was created to obtain quantitative apparent-height judgments from naive observers. We assume that if the magnitude of vertical motion is not related to phenomenal height. then this is still another reason not to restrict the definition of the SKE to picture-plane rotations of two-dimensional patterns.

In addition, we investigated two other factors. First, it has often been reported that apparent height of SKE cones varies as a function of eccentricity (Mefferd \& Wieland, 1968; Musatti, 1955; Robinson. Piggins, \& Wilson, 1985; Wilson, Robinson, \& Piggins, 1986; Zanforlin, 1988). We here sought to confirm this relationship using a new procedure for assessing apparent height. Second, we compared the perceived height of SKE displays with depth judgments made while viewing static images of these patterns.

\section{Method}

Subjects. Twelve University of Virginia undergraduates, 6 women and 6 men, were recruited from an introductory psychology course and received partial credit for their participation. None of them had participated in previous SKE experiments.

Stimuli. Seven KDE stimuli varying in the objective height simulated were used to train subjects with the procedures before the presentation of the SKE test stimuli. In all cases, the tilt of the KDE cone was constant $\left(\alpha=36.9^{\circ}\right)$, which produced a foreshortening of $20 \%$. Eccentricity values were $0,0.2,0.4,0.8,1.2$, and 1.6 corresponding to heights of $0,0.17,0.33,0.67,1.0$, and 1.32 diameters of the base. Note that when eccentricity $(\mathrm{e} / \mathrm{r})$ exceeded 0.8 , the tip of the virtual cone extended beyond the base, and the contours were partially occluded as if they resided on an unseen, opaque cone. Such a stimulus is depicted to the right in Figure 5. SKEs were created that varied in eccentricity $(0,0.32,0.64$, and 0.96$)$. All stimuli subtended $1.3 \mathrm{~cm}$ at their base. consisted of four circular contours. and rotated at $0.65 \mathrm{~Hz}$.

The apparent motion of a traditional SKE stimulus can be decomposed into two simultaneous sinusoidal motions: a horizontal ( $x$-axis) and a vertical ( $y$-axis) motion. Here three motion conditions were introduced: (a) a turntable-producible SKE whose center (of base) was displaced in the direction of its tip by 0.8 radii from the turntable center (i.e.. computer simulation of full $x$-and $y$-axis motions). (b) a display with equivalent $x$-axis motions and with a vertical motion component that was reduced by one half, (c) a display with $x$-axis motion only (i.e.. the contours translated horizontally back and forth with sinusoidal velocity functions). Unlike fully rotating displays. eccentricity is not constant for SKE stimuli having reduced vertical motions. but rather it changes continuously and only reaches its maximum during the left-most and right-most points in their motion. The motion of KDE stimuli was similarly constructed: The base of the cone moved sinusoidally with (a) both $x$ - and $y$-axis components around a point displaced away from its tip by 0.8 radii from its center, (b) full $x$-axis and only one half the $y$-axis motion. (c) full $x$ axis and no $\mathrm{l}$-axis motion.

In addition to these moving contour displays, stationary versions were created from single frames of the SKE and KDE stimuli. Although this introduces a contradiction in terms. we refer to these stimuli as stationary SKE and stationary KDE displays. They con- sisted of nested contours with the same eccentricity values: four stationary KDE patterns $(\mathrm{e} / \mathrm{r}=0,0.4,0.8$, and 1.2$)$ and four stationary SKE patterns $(e / r=0,0.32,0.64$, and 0.96$)$. The purpose of including these stationary displays was to assess the magnitude of depth judgments that their pictorial cues might evoke.

Two synchronized height measures were used: the top view of a stereo pyramid and a separate cross-section of that pyramid. Their heights could be adjusted together by the observers while they viewed the ongoing displays. The stereoscopic pyramid was located $3 \mathrm{~cm}$ to the left of the test stimulus and consisted of a line drawing of a rectangular pyramid viewed from the top. The contours were green just as was the stimulus. A second pyramid was drawn on top of it in red. Observers would view the display anaglyphically through red and green filters that were inserted in the viewing box. By pressing one key for each direction, they could change the disparity between the two pyramids, such that when fused the impression of a pyramid shrinking or growing in height resulted. The other measure consisted of a line about $3 \mathrm{~cm}$ to the right of the stimulus. Synchronized with the anaglyphic pyramid, this line would grow into a triangular crosssection of the pyramid on the left, pointing at a $45^{\circ}$ angle toward the upper right corner of the screen. The triangular cross-section and test stimuli were drawn in green and, thus, were viewed only by one eye.

Design. A within-subjects design was used. Subjects were first trained on KDE stimuli and then had to judge the height of SKEs based on this training. For contours on an actual stationary cone, eccentricity and aspect ratio geometrically determine the height of the cone under the assumption that the cone is of a regular circular form. The experiment was divided into eight blocks. Block $1: \mathrm{Ob}-$ servers were trained on stationary KDEs. During training, they were given feedback as to whether their setting was too high, too low, or correct (defined as a setting that was within $8 \%$ of the actual height of the projected object). Training continued until two criteria were reached: Three consecutive stimuli had to be judged correctly (i.e., within $8 \%$ of the actual projected heights). and observers must have completed at least six triais; (i.e., if they met the first criterion in five or fewer trials, then they continued until six training trials had been completed). If the first criterion had not been met after 20 trials, the subject's data were not included in the analysis. Block 2: Observers were tested on four static SKEs. Here there is no unique geometrical solution. Block 3: Observers were then given four test trials without feedback with stationary KDE patterns followed by four more test trials with the SKE stationary patterns (Block 4). After these tests with the stationary displays, the same procedure was repeated for moving stimuli: (Block 5) training on seven moving KDEs; (Block 6) testing on the four moving SKEs differing in eccentricity (three times each with a different level of $y$-axis motion in random order); (Block 7) seven test trials with the KDE stimuli without feedback; (Block 8) retesting on the four SKE displays three times each with a different level of $y$-axis motion in random order.

To reiterate, observers were given training only with the static and moving KDE patterns. These displays presented a sufficient amount of information for a geometrical determination of their height. The purpose of this training was primarily to reduce the variability in observers' responses to the SKE patterns.

Procedure. Except for the anaglyphic viewing procedure, the experimental situation (graphics screen and viewing box) was the same as in Experiment 1. The experimenter had a separate monitor to control the experiment and to provide feedback (in training blocks only). Observers were asked to adjust the probe triangle or pyramid until they thought that it matched the height of the training stimulus. Feedback indicated whether the probe was set appropriately or was set too shallow, much too shallow, too high, or much too high. On each training trial, this procedure was repeated until the probe's height was within $8 \%$ of the actual stimulus height. 
For the moving SKEs, observers were asked to look at the stimulus until they could see it as a cone pointing toward them and accordingly only stretch the probe upward. Within each block, stimuli were presented in random order. Subjects were introduced to both height measures but were free to attend to only one of them if they liked. Postexperimental reports suggested that most subjects relied heavily on the adjustable triangle.

\section{Results and Discussion}

It was hypothesized that turntable-producible motions are not requisite for SKE. In accord with this hypothesis, the amount of $y$-axis motion for moving SKE stimuli had no effect on perceived height, $F(2,46)=1.0, p>.35$, and the mean height settings for the three levels of $y$-axis motion were essentially identical at all eccentricities.

Height judgments for all other conditions are shown in Figure 9. It was found that eccentricity was a good predictor for judged height in stationary as well as in moving stimuli. As was expected because of the training given, KDE heights were judged accurately in both motion and stationary conditions. SKE heights were judged to be considerably smaller in stationary as opposed to moving conditions.

The following results were obtained for SKE stimuli: Motion yielded increased height judgments, $t(11)=5.2, p<.001$. Judged height was $40.1 \%$ lower for stationary SKEs. Figure 9 shows that the larger the eccentricity of both static and moving SKEs, the taller they were judged to be, $F(3,69)=263.84, p$ $<.0001$. Judged height and eccentricity were highly correlated ( $R=.85$ for moving SKEs and $R=.91$ for static SKEs). As noted previously, this strong positive correlation between eccentricity and height for moving SKE patterns has been found in a number of previous studies. A quadratic regression function fit the moving SKE height judgments well $\left(R^{2}=\right.$ $.78): \mathrm{h}^{\prime} / 2 \mathrm{r}=2.5 *(\mathrm{e} / \mathrm{r})-1.3 *(\mathrm{e} / \mathrm{r})^{2}$. Zanforlin $(1988)$ proposed a model for perceived SKE height in which $h^{\prime}=2 r$ $+\mathrm{e}$. In our experiment, judged heights for moving SKEs fit this model quite well for large eccentricities but were lower than predicted for small eccentricity values.

For KDE, apparent height judgments were analyzed for those trials that did not involve feedback; unlike the findings for SKE, motion did not influence these judgments, $t(11)=$ $1.45, p>.1$. For both static $(R=.93)$ and moving $(R=.96)$ displays, objective height and eccentricity were strongly correlated with judged height. Comparing the judged with the objective mean height for each stimulus (eliminating the 0 eccentricity stimulus) led to an average unsigned estimation error of $8.3 \%$ for static KDEs and $7.8 \%$ for moving KDEs.

In summary, the main hypothesis assessed in this experiment was confirmed: A single-axis transformation alone-in this case, along the $x$-axis-suffices to evoke stereokinesis. Perceived height for single-axis motions were no smaller than those evoked by the more traditional turntable method that necessarily includes both $x$-axis and $y$-axis transformations. In addition, eccentricity was found to be strongly related to height judgments for both moving and static SKE patterns. Finally, the difference found in height judgments for the moving versus static patterns suggests that the height per- ceived in moving SKE displays is not inferred solely from static pictorial cues. The static pattern, of course, always looked two dimensional but, when told to view it as a threedimensional cone, observers were able to make reliable depth judgments. However, the magnitude of these judgments fell far short (about $40 \%$ ) of the height perceived in the moving SKE displays.

\section{Experiment 7}

In this final study, we extend the generality of our SKE definition from contour motions to those of surfaces. We created a dense pattern of dots with a texture gradient equivalent to the eccentricity of an SKE cone. This pattern was moved in accordance with our general SKE definition: Points moved according to their assignment in $z$-depth, but the whole pattern maintained orientation stability. Individual observers viewed static or moving versions of this event and made phenomenal reports.

\section{Method}

Subjects. Twelve University of Virginia undergraduates $(6$ women and 6 men) were paid for their participation in this and other unrelated experiments that were being conducted in our lab. None of them had been previously exposed to SKE displays.

Stimuli. An SKE display was created, the surface of which consisted of a dense gradient of approximately 400 dots. This surface could be thought of as an SKE cone consisting of 400 virtual contours with one visible dot on each (at a random location on each contour). The resulting density gradient increased continuously toward the cone's apex, which had an eccentricity of 0.4 . The display contained equal amounts of $x$ - and $y$-motion but was not turntable producible because the virtual contours maintained orientation stability. The base of the display was stationary. Each frame of the display was generated on a Sun $3 / 60$ color graphics workstation and then recorded frame by frame onto $3 / 4-\mathrm{in} .(1.9-\mathrm{cm})$ videotape. The animated display was played back at a rate of 30 frames per second $(60 \mathrm{~Hz}$ interlaced) on a Sony Trinitron color monitor (diagonal screen width of $34.5 \mathrm{~cm}$ ). Viewing distance was about $100 \mathrm{~cm}$, the display subtended $6.5 \mathrm{~cm}$, and rotation was $0.50 \mathrm{~Hz}$. A second stimulus consisted of a video display showing one static frame of the same stimulus.

Design and procedure. A between-subjects design was used such that each observer saw only one stimulus. Instructions were identical to those of Experiment 5: Spontaneous reports were obtained as was the time until a three-dimensional organization occurred. Stimuli were presented in a room that was almost completely dark, and observers were asked to cover up one eye with their hand. They were tested individually. Nine observers were tested in the moving condition and 3 in the static condition.

\section{Results}

Moving random-dot SKEs were always perceived as threedimensional objects: $77.8 \%$ of observers reported cones pointing toward them, $11.1 \%$ saw a tunnel-like object, and $11.1 \%$ reported a different three-dimensional object (e.g., a footballlike object). On average, subjects mentioned the perception of three dimensionality after $8.72 \mathrm{~s}$. After viewing the static random-dot display for at least $1 \mathrm{~min}$, none of the 3 observers 


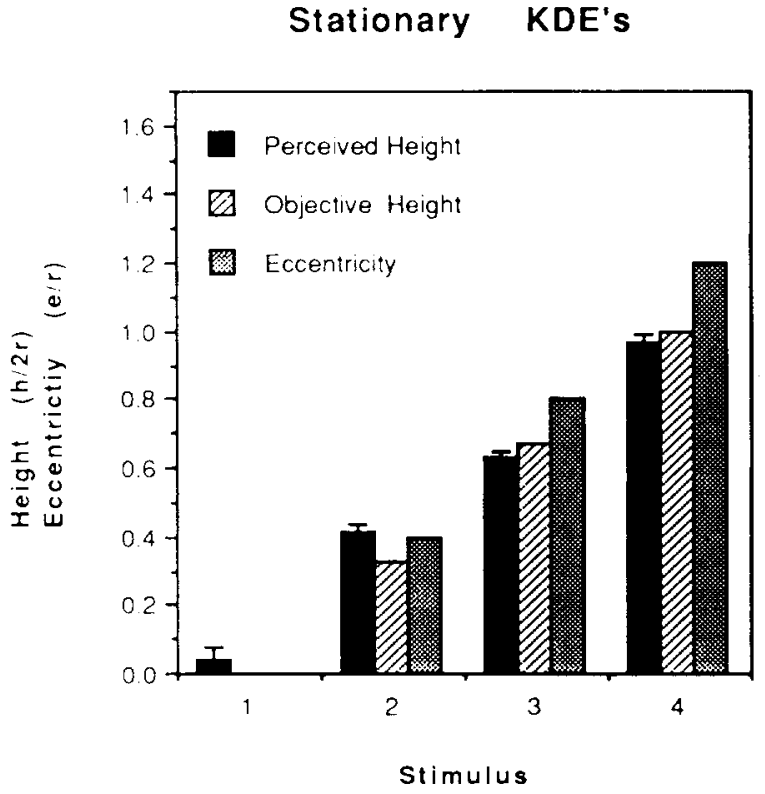

Moving KDE's

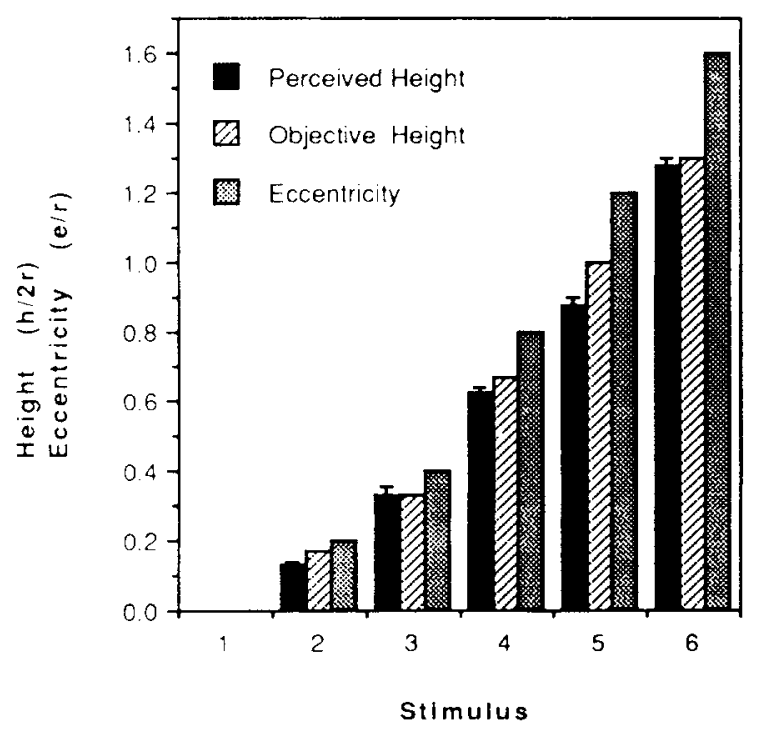

Stationary SKE's

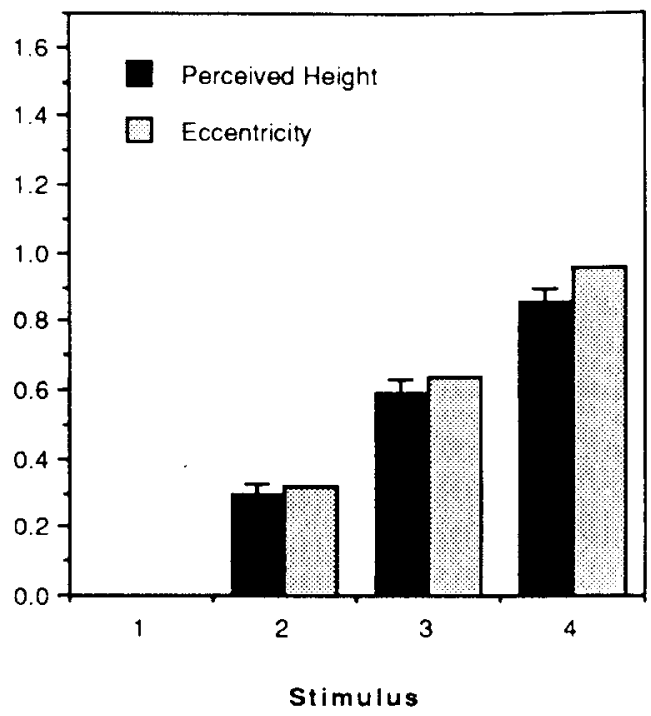

Moving SKE's

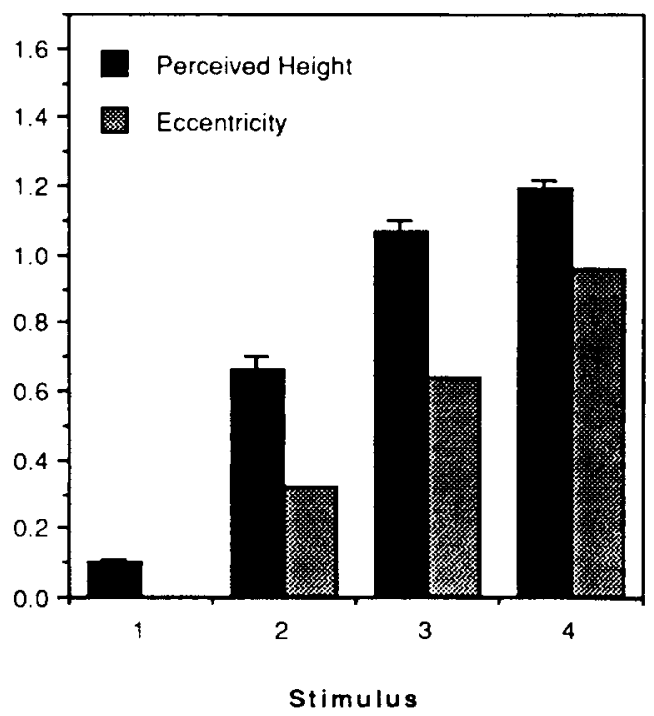

Figure 9. Means for perceived height obtained in Experiment 6. (There were four stationary and six moving kinetic depth effect [KDE] stimuli varying in objective height and eccentricity, the values of which are plotted along with those for perceived height. Note that unlike height $[h / 2 r]$, eccentricity is based on the radius $[\mathrm{e} / \mathrm{r}]$ rather than the diameter. There were four stationary and moving stereokinetic effect [SKE] stimuli that varied in eccentricity from 0 to 0.96 radii of the base. Because these displays have no objective height, here only perceived height judgments are plotted along with eccentricity values.)

reported phenomenal depth. (We stopped running observers in the static condition because it was clear that no one was going to report depth in this display. We could see none ourselves.)

\section{General Discussion}

The perception of SKE displays is intriguing in at least two respects. First, the stimulus conditions for the SKE are almost equivalent to those that prevail for small rotations of rigid objects, and the percepts that are evoked under both of these conditions are quite similar. We find this similarity to be suggestive of how the visual system extracts depth relationships when viewing small rotations in everyday situations. Second, the stimulus conditions for the SKE are not sufficient to geometrically specify object-relative depth, and yet people perceive a specific depth in these displays with as much 
consistency as when viewing KDE displays where depth percepts are geometrically supported. These related aspects of the SKE are discussed in turn.

The similarity between the stimulus bases for the SKE and the $\mathrm{KDE}$ under small rotations point to a well-known geometrical fact: When an object is observed to undergo small rotations-either because it has rotated relative to a stationary observer or because the observer has moved relative to it in some direction other than the line of sight-the motions that occur between points at different $z$-depths are quite large relative to those that occur between points initially at the same $z$-depth. In fact, the instantaneous relative velocity for points at the same $z$-depth is exactly 0 .

For purposes of exposition, consider what happens when an observer views the configuration of three points shown in Figure 10. Here the two far points have the same $z$-depth, whereas the third point is nearer in depth. Rotating this
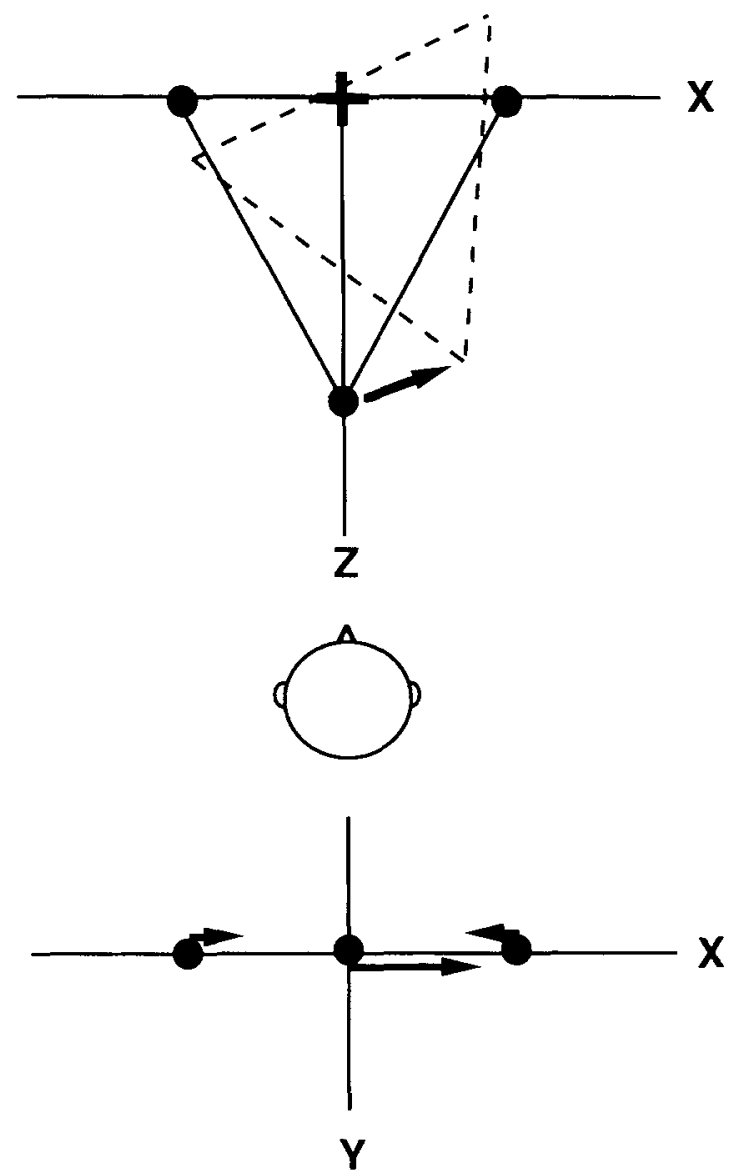

Figure 10. At the top are depicted three points being observed by an observer. (The situation is drawn as if viewed from above. Two points fall on a line normal to the observer's line of sight, the $x$-axis, and the third is equidistant from the other two and nearer to the observer in the $z$-axis direction.) When this configuration rotates counterclockwise, the motions projected to the observer are as depicted in the bottom panel. (The far points move toward each other as a cosine function of the angle of rotation and the distance between them, whereas the near point moves to the right as a sine function of the angle of rotation and its $z$-axis distance to the point of rotation.) configuration around a $y$-axis at the midpoint of the configuration's base yields a projected displacement of the near point that is a sine function of the angle of rotation, whereas the projected displacements of the far points follow cosine functions. See the bottom arrows in Figure 10. As we all know, the derivative of the sine function for small angles is enormous relative to the derivative of cosine values for the same angles. Consider that for this depicted configuration a $15^{\circ}$ rotation displaces the near point by $26 \%$ of its objectrelative depth, whereas each one of the far points moves an amount that is only $3 \%$ of half the distance between them. Moreover, the near point is displaced by a full half of its object-relative depth after a $30^{\circ}$ rotation, yet at this observerrelative change in slant the two far points move toward each other by only $13 \%$ of the distance between them.

As regards theories for how people recover depth from motion, there are consequences of these geometrical facts considered in conjunction with an appreciation of the perceptual response to the SKE. As is always true when viewing an SKE display, when observing small rigid-object rotations, there may be no effective information available to specify the amount of rotation that has occurred; thus, the magnitude of object-relative depth cannot be determined geometrically. Again consider the situation depicted in Figure 10. Because the magnitude of the near point's displacement is determined by two variables-amount of rotation and its object-relative $z$-depth-this point's projected displacement does not in itself specify either. That is, this stimulus change is ambiguous. On the other hand, the relative displacement of the two far points does, in principle, specify the magnitude of rotation because, being initially normal to the line of sight. the distance between these points is given (up to an absolute distance-relative scale factor). In other words, the amount of foreshortening between points at the same object-relative depth does specify the degree of rotation of the object. Once that is specified, object-relative depth is no longer ambiguous. However, when rotations are small, the relative displacement of the points at the same object-relative depth is barely detectable and thus cannot be of much perceptual utility in specifying the magnitude of rotation. For both SKE and KDE displays in which there are small rotations, the magnitudes of rotation and object-relative depth are confounded. Be that as it may, the perceptual system assigns definite depth values anyway.

Consider, for example, formal models of optical flow that rely on instantaneous velocity fields such as that of Koenderink (1986). These models do not detect transformations resulting from changing slant for points on surfaces normal to the line of sight because these motions are instantaneously equal to 0 . Therefore, instantaneous flow models need to obtain additional information about how much rotation has occurred, and this is typically done by acquiring information about observer-relative displacement. In the SKE, there is information that the object is rotating but none about the magnitude of this transformation; for this reason, the magnitude of $z$-depth is unspecified.

Considered from this perspective, the motions of the SKE are sufficient to allow the extraction of sequential information about $z$-depth but not the cardinal depth values, and, in addition, the order of points is unsigned. (The stimulus bases for the KDE under orthographic projection do provide size- 
relative cardinal depth values but not sign.) Yet the visual system assigns a specific $z$-depth interpretation to SKE displays with very little within- or between-observer variability. Clearly. the visual system is doing something that is not entirely explicable in terms of a canonical geometrical analysis of the stimulus.

We propose that when presented with small rotations, the visual system is able to extract only sequential information about object-relative depth from the salient relative motions that are present. Depth order is then assigned either from perspective, occlusion, or observer motion information when available or from inherent perceptual biases in the case of reduced displays. In the latter case, depth order will typically reverse spontaneously. Finally, because information specifying the magnitude of rotation is absent or too small to be reliable, the assignment of a depth value cannot be achieved through an appropriate geometrical analysis of the stimulus conditions. However, the perception of ordinal depth alone, without a concurrent perception of the depth magnitude, is not a phenomenal possibility; thus, the perceptual system must derive a depth interpretation by some means. SKE displays are perceived to have a height that is proportional both to the magnitude of the pattern's relative motions and to the width of its base, the ratio of which defines the display's eccentricity. For small rigid-object rotations, we suspect that depth assignment is made in a manner similar to the processing of the SKE: Depth is specified by a function that relates the perceived width of the object to the magnitude of its relative motions. That is, object-relative depth is strongly related to the magnitude of eccentricity $(\mathrm{e} / \mathrm{r})$.

Why should the depth perceived in SKE displays be precisely what it is? Other than the qualitative account given previously, we cannot at this time offer a more precise rationale for why the perceived height of SKE displays is the particular function of size-relative eccentricity that it was found to be. Zanforlin (1988) proposed a model for determining the height of SKE cones that fit his and Musatti's (1955) data quite well. This model, however, can be applied only to two-dimensional patterns that are rotated in the picture plane. It cannot be applied to stimuli that cannot be produced by the traditional turntable method. As was found in Experiment 6 , patterns with linear relative motions are perceived as having the same height as those manifesting full rotations. Moreover, compared with traditional SKE cones, similar ratings for amount of depth, compellingness, and rigidity were found for displays having elliptical, square, triangular, and polygon contours that did not rotate but rather maintained orientation stability. Zanforlin's model requires that motions along rotating contours be detected; thus, it cannot account for such displays and must be rejected. Musatti (1924) postulated a threshold account in which perceived height was predicted to be the minimal value consistent with the threshold for discriminating between circles and ellipses. That is, perceived height was determined by assuming that the apparent cone had the least amount of depth consistent with the following conditions: (a) displayed eccentricity, and (b) a rotation equivalent to the maximum slant that would project an ellipse not noticeably different from a circle. Musatti (1928-1929) later rejected this account when he assessed the discrimination threshold between static circles and ellipses and determined that it occurred at a slant of over $20^{\circ}$. Rotations of such large magnitudes would result in perceiving cones that were far too shallow. Musatti's methods were not very sensitive, and, as our Experiment 4 showed, much smaller slants are detectable at least in the context of our moving displays. More to the point, however, Musatti did not define eccentricity relative to the size of the display $(\mathrm{e} / \mathrm{r})$. SKE patterns having equivalent eccentricities but different sizes evoke equivalent object-relative depths $(\mathrm{h} / 2 \mathrm{r})$. This fact is incompatible with Musatti's threshold proposal.

\section{Conclusion}

The SKE typically has been thought of as a kind of depth illusion, and in a strict sense of the term it is. SKE displays can be produced by rotating certain two-dimensional patterns on a turntable and, when observed, they evoke the perception of compelling three-dimensional objects. Alternatively, the actual stimulus bases for the SKE are strikingly similar to those that occur when a rigid object is observed to undergo small rotations. In these latter cases, the motions of points separated in object-relative depth will move considerably, whereas those that fall on planes normal to the line of sight will manifest negligible motions. We have defined the stimulus bases for the SKE as the presence of the former motions and a complete absence of the latter. This definition is consistent with the motions observed in traditional SKE displays, but it is far more general and allows for the creation of SKE patterns that could not be produced by the turntable method. The studies reported here show that displays created in accordance with our definition, but not producible by the turntable method, evoke compelling depth percepts equivalent to those seen in traditional turntable displays. For example, it was shown that equivalent virtual objects are perceived when the contours of an SKE object are simply translated relative to each other as when the entire pattern is rotated.

SKE displays are perceived to have a definite height that varies little within or between observers. This is perplexing, but also we feel it is of considerable significance for understanding how the perceptual system derives depth from small rotations in everyday contexts. In such situations, the visual system is presented with compelling information about sequential depth relations but is given insufficient information about depth magnitudes. Clearly, the perceptual response in these circumstances goes beyond a purely geometrical extraction of depth from the prevailing stimulus conditions.

We propose that when small rotations of $15^{\circ}$ or less are being observed, the perception of depth magnitudes from monocular motion information does not follow a canonical geometrical analysis of the stimulus conditions. Only objectrelative motions are used because they are the only motions of sufficient salience to be of perceptual utility. Observerrelative motions are barely detectable at $15^{\circ}$ and fall below threshold at smaller angles of rotation. Without a precise appreciation for these observer-relative motions, the amount of object rotation cannot be determined, and depth magnitude cannot be computed from object-relative motions alone. That 
definite depths are observed under these conditions implies that the perceptual system is using inherent biases or assumptions.

\section{References}

Hildreth, E. C. (1984). Computations underlying the measurement of visual motion. Artificial Intelligence, 23, 309-354.

Koenderink, J. J. (1986). Optic flow. Vision Research, 26, 161-180.

Mefferd, R. B., \& Wieland, B. A. (1968). Perception of depth in rotating objects: 7 . Influence of attributes of depth on stereokinetic percepts. Perceptual and Motor Skills, 27, 1179-1193.

Musatti, C. L. (1924). Sui fenomeni stereocinetici. Archivio Italiano de Psicologia, 3, 105-120.

Musatti, C. L. (1928-1929). Sulla percezione di forme di figura oblique rispetto al piano frontale. Rivista di Psicologia, 25, 1-14.

Musatti, C. I. (1955). La stereocinesi e il problema della struttura dello spazio visibile. Rivista di Psicologia, 49, 3-57.

Musatti, C. I. (1975). Stereokinetic phenomena and their interpretation. In G. B. Flores d'Arcais (Ed.), Studies in perception. Festschrift for Fabio Metelli (pp. 166-189). Milan: Martello-Giunti.
Robinson, J. O., Piggins, D. J., \& Wilson, J. A. (1985). Shape height and angular movement in stereokineses. Perception, 14, 677-683.

Rock, I. (1983). The logic of perception. Cambridge, MA: MIT Press.

Wallach, H. (1935). Über visuell wahrgenommene Bewegungsrichtung. Psychologische Forschung, 20, 325-380.

Wallach, H., \& Centrella, N. M. (1990). Identity imposition and its role in a stereokinetic effect. Perception \& Psychophysics, 48, 535542.

Wallach, H., \& O'Connell, D. N. (1953). The kinetic depth effect. Journal of Experimental Psychology, 45, 205-217.

Wallach, H., Weisz, A., \& Adams, P. A. (1956). Circles and derived figures in rotation. American Journal of Psychology, 69, 48-59.

Wilson, J. A., Robinson, J. O., \& Piggins, D. J. (1986). Apparent height of a stereokinetic cone is decreased by departure from circularity. Perception, 15, 399-403.

Zanforlin, M. (1988). The height of a stereokinetic cone: A quantitative determination of a 3-D effect from 2-D moving patterns without a "rigidity assumption." Psychological Research, 50, 162172.

Received January 7, 1991

Revision received May 20, 1991

Accepted May 20, 1991

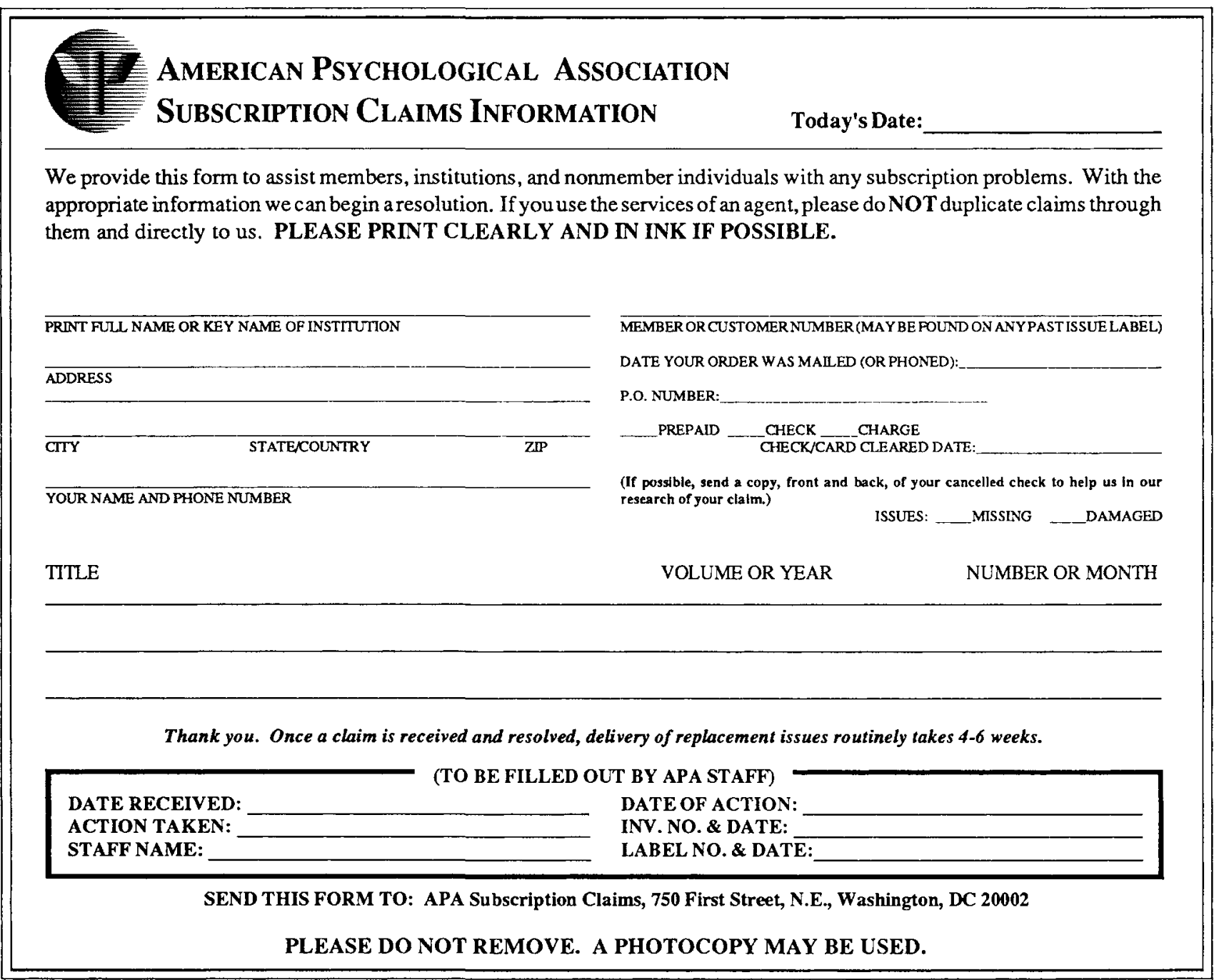

